\title{
Robotic Router Formation in Realistic Communication Environments
}

\author{
Yuan Yan and Yasamin Mostofi, Member, IEEE
}

\begin{abstract}
In this paper, we consider the problem of robotic router formation, where two nodes need to maintain their connectivity over a large area by using a number of mobile routers. We are interested in the robust operation of such networks in realistic communication environments that naturally experience path loss, shadowing, and multipath fading. We propose a probabilistic router formation and motion-planning approach by integrating our previously proposed stochastic channel learning framework with robotic router optimization. We furthermore consider power constraints of the network, including both communication and motion costs, and characterize the underlying tradeoffs. Instead of taking the common approach of formation optimization through maximization of the Fiedler eigenvalue, we take a different approach and use the end-to-end bit error rate (BER) as our performance metric. We show that the proposed framework results in a different robotic configuration, with a considerably better performance, as compared with only considering disk models for communication and/or maximizing the Fielder eigenvalue. Finally, we show the performance with a simple preliminary experiment, with an emphasis on the impact of localization errors. Along this line, we show interesting interplays between the localization quality and the channel correlation/learning quality.
\end{abstract}

Index Terms-Networked robots, realistic communication channels, robotic router formation.

\section{INTRODUCTION}

$\mathbf{T}$ HE vision of a multiagent robotic network cooperatively learning and adapting in harsh unknown environments to achieve a common goal is closer than ever. The fundamental problem that arises in such networks is maintaining connectivity and ensuring a robust flow of information [1]-[5]. Along this line, robotic router problems have started to receive considerable attention in recent years [6]-[8]. In such problems, a transmitting node needs to maintain its connectivity to a receiving node over a large distance. Since the receiving node may need to get far due to possible exploratory missions, direct transmission may

Manuscript received July 5, 2011; revised October 29, 2011; accepted February 9, 2012. Date of publication April 12, 2012; date of current version August 2, 2012. This paper was recommended for publication by Associate Editor N. Y. Chong and Editor D. Fox upon evaluation of the reviewers' comments. This work was supported in part by the National Science Foundation (NSF) CAREER award 0846483 and in part by the NSF award 0812338 . Part of this paper was presented at the 2010 Military Communications Conference 2010.

The authors are with the Department of Electrical and Computer Engineering, University of California, Santa Barbara, CA 93106, USA (e-mail: yuanyan@ece.ucsb.edu; ymostofi@ece.ucsb.edu).

Color versions of one or more of the figures in this paper are available online at http://ieeexplore.ieee.org.

Digital Object Identifier 10.1109/TRO.2012.2188163 not be possible. Therefore, a number of robotic routers can be used to ensure robust communication between the two nodes. The routers will reconfigure themselves constantly in order to optimize the flow of information. The fundamental question is then as follows: Given specific transmitter/receiver locations and environmental/communication constraints, what is the optimum configuration of the routers and how can it be reached?

In the robotics and control community, algebraic-graph approaches attracted considerable attention to solve this problem. In [2], the Fiedler eigenvalue is taken as a metric for the connectivity of a state-dependent graph and semidefinite programming is used to maximize it, subject to minimum distance constraints. In [3], a decentralized algorithm which is based on supergradient and decentralized computation of Fiedler eigenvector is proposed. Similarly, a potential function is defined in [4], using spectral properties of the Laplacian matrix, in order to optimize connectivity, while $k$-connectivity constraints are imposed in [5]. In [6], the ideas from [2], [3], and [5] are integrated to design a centralized control law for robotic routers. While the Fiedler eigenvalue is one measure of graph connectivity, it is rather a high-level measure, i.e., it does not measure the communication reception quality. In [9], a spring-damper model is adopted to enforce the connectivity of a robotic operation, in an outdoor environment. A spring-damper model, on the other hand, still requires designing a function that translates the link quality to proper forces. In [7], two robotic router algorithms, which are known as user trajectory algorithm and adversarial user trajectory algorithm, are proposed. More relevant communication-oriented metrics have also been utilized. In [8], channel capacity notions are used to avoid RF disturbances, assuming disk models for communications. While this is more relevant to the quality of a link, capacity is an upper bound on the performance, rather than a measure of the current communication quality. In [10], the mobility control problem is solved by minimizing the communication energy cost, while in [11], the mobility control problem is investigated with the goal to maximize the lifetime of the network. All the aforementioned work, however, assumes a priori known disk or path-loss-only models for communication.

In the wireless sensor network community, optimal positioning of wireless sensors (or relays) has been studied [12], [13]. Similarly, in the communication literature, there has recently been a considerable interest in cooperative communication, typically with the assumption of one relay node [14], [15]. While communication-related metrics are used in these problems, most of the work from the communication or networking community is not concerned with the control of motion, i.e., it is not on how a number of routers can plan their motions to optimally position themselves. 
In summary, there are three motivations for the work that is presented in this paper. First, most work that is concerned with motion planning in robotic routers does not use a metric that is communication-oriented. Second, the papers that use more communication-oriented metrics are typically not concerned with motion planning and optimization. Finally, a proper motion optimization requires an assessment of the link qualities at unvisited locations, which is something that is currently missing in robotic router positioning or motion optimization.

More specifically, in this paper, we are interested in the robust operation of a robotic router network in realistic communication environments, which will naturally experience fading. Most aforementioned work on robotic routers only considers simplified homogeneous disk models to model communication links, without showing how the details can be learned in practice. As such, their performance can degrade considerably in realistic communication environments. In order to ensure reliable router connectivity in fading environments, each router needs to predict wireless channels in positions it has not yet visited and build objective functions that properly integrate both communication and motion goals. Building proper models for wireless channel prediction in fading environments is a challenging task. In [16] and [17], a framework was developed for probabilistic channel prediction in robotic networks. In this paper, we furthermore show how the aforementioned channel prediction framework can be integrated with robotic router optimization to ensure an effective flow of information in fading environments. It is, therefore, our hypothesis that without properly integrating communication and motion issues, robotic router optimization will not be possible.

Before developing the proper framework for the optimization of a robotic router network, a proper metric of performance needs to be defined. For instance, if the transmitting robot sends a number of bits to the receiving robot, what percentage will be received accurately? This is a metric that is used extensively in the communication literature. In other words, the effectiveness of the routers can be measured by the bit error rate (BER) of the end node. BER measures the probability that a bit gets flipped, i.e., it is received in error, and is one of the most important measures of digital communication performance [18], [19]. In this paper, we are, therefore, interested in optimizing the robotic router network by using the BER as our connectivity metric. Then, the optimum configuration of the nodes is the one that results in the smallest possible BER at the receiving node. We show how this metric results in a different robotic configuration, with a considerably better performance, as compared with maximizing the Fiedler eigenvalue.

In summary, the contributions of this paper are 1) integrating probabilistic channel prediction models with robotic router optimization, in order to ensure robust operation in environments that experience path loss and fading, and 2) showing the impact of power constraints on the optimization of robotic routers, considering both communication and motion costs. Moreover, we develop our robotic router optimization framework by using BER as the performance metric.

The rest of this paper is organized as follows. Section II describes our problem setup and connectivity metric. In Section III, we consider robotic router optimization in path-loss environments, using BER as a performance metric. In Section IV, we extend our analysis and design to fading environments, where we develop a probabilistic framework for robotic router optimization. In Section V, we consider optimization of robotic routers under power constraints, by taking into account both communication and motion costs. In Section VI, we present our preliminary experimental results to verify the effectiveness of our framework and show the impact of localization errors. Interesting interplays between the impact of localization errors and channel learning errors are also discussed. We conclude in Section VII.

\section{Problem SetuP}

Consider a team of $m$ robots that are spatially distributed in a given environment to perform a task jointly. Let node 1 indicate a transmitting node (TX) that needs to send information to node $m$, i.e., the receiving node ( $\mathrm{RX})$, which can be considerably far from node 1 . The rest of the nodes will act as robotic routers by relaying the information, i.e., they spatially position themselves such that the flow of information is maximized from the transmitting node to the receiving one. Our goal is to find the optimum configuration in realistic communication environments and control the motion of the routers such that they converge to it (given stationary TX/RX). In this paper, we use the terms "robot" and "node" interchangeably to represent all the agents, including the transmitter, the receiver, and any router. We use the term "router" to specifically indicate a robotic router. Furthermore, we consider stationary TX and RX nodes, in order to focus on the optimum configuration and motion planning of the routers with realistic wireless channels and metrics. We, however, note that our framework can be extended to accommodate mobile TX and/or RX.

\section{A. Link Performance Metric}

As mentioned earlier, in this paper, we are interested in the robotic router optimization by using the end-to-end BER as the performance metric. BER characterizes the probability that a bit arrives in error (flipped) at the receiver and has been used extensively to characterize the performance in the communication literature [18]. Another related fundamental parameter that characterizes the performance of a communication channel is the received signal-to-noise ratio (SNR). The received SNR is defined as the ratio of the received signal power divided by the receiver thermal noise power. The instantaneous received SNR directly impacts the BER and, as a result, the reception quality. In general, there are three time-scales that are associated with the spatio-temporal changes of the channel quality and, therefore, the received SNR. The slowest dynamic, path loss, is associated with the signal attenuation due to the distance-dependent power fall-off. Depending on the environment, there could be a faster variation, referred to as shadow fading (or shadowing), which is due to the blocking objects. Finally, multiple replicas of the transmitted signal can arrive at the receiver due to the reflection from the surrounding objects, resulting in even a faster variation in the received signal power called multipath fading. The 
left side of Fig. 4 shows a simulated wireless channel, with the three underlying dynamics. As can be seen, the channel can vary considerably. In most robotic literature, a priori known communication disk models are assumed when motion planning. As can be seen from the left side of Fig. 4, planning only based on even the path-loss component can result in a severe performance degradation in practice. Therefore, in this paper, we are interested in motion planning and optimization of router configuration, as well as characterizing the implication of BER for motion planning and control, in realistic communication environments.

BER shows how the received SNR, modulation, channel coding, and other transmission parameters affect the performance [18]. As a result, a general expression for BER does not exist. Consider communication between two nodes. Let $b$ and $\hat{b}$ represent a transmitted bit (as part of a transmitted packet) and its reception (after passing through a decision device), respectively. Then, BER is defined as $P_{b}=\operatorname{Prob}\{\hat{b} \neq b\}$. For an additive white Gaussian noise channel (AWGN), BER can be represented or finely approximated by a Q function or an exponential function. In [18], a general approximation (an upper bound) for the BER of an MQAM (M-ary Quadrature Amplitude Modulation) transmission is derived as follows: ${ }^{1}$

$$
P_{b} \approx 0.2 \exp \left\{-\frac{1.5}{M-1} \gamma\right\}
$$

where $M$ is the modulation constellation size, and $\gamma$ is the received SNR. This approximation is tight (within $1 \mathrm{~dB}$ ) for $M \geq 4$ and $0 \mathrm{~dB}<\gamma<30 \mathrm{~dB}$. In this paper, we use this approximation to characterize the BER of each reception.

\section{B. Communication Topology}

In this paper, we assume a multihop topology for the robotic router network, i.e., each node relays the information that is received from its previous node, to the next one, until it reaches the RX node. This topology can be built by using a route discovery scheme from the ad hoc routing literature, such as dynamic source routing (DSR) [20] or ad hoc on-demand distance vector routing (AODV) [21]. Once a valid route is found, the transmitter will receive a packet that contains a summary of the sequence of loop-free hops to the receiver, which can be used to deliver messages. See [20] and [21] for more details. Each robot then broadcasts the received message once it receives it from its previous node. This process continues until the message reaches the final destination (receiving node). Our study then focuses on co-optimizing the motion planning and communication performance metrics of this route. In other words, similar to other work on robotic router motion planning, we do not focus on the layer that corresponds to routing and topology discovery as there is a rich body of work on it in the ad hoc routing literature. Instead, we focus on the integration of communication issues (physical layer) with motion planning (application layer).

\footnotetext{
${ }^{1}$ MQAM is a common class of modulation in the communication literature.
}

While we assume a multihop route in this paper, our study can also be extended to other topologies. For instance, in [1], we considered a diversity-based topology, in which a node receives multiple copies of the transmitted information from different routers. The framework of this paper can be extended to such topologies as well. It should be noted that in this paper, we are not assuming a highly dense network (such as swarms). Thus, we are not concerned with multiple access issues (such as interference among the nodes) that can arise when the number of nodes, sharing the given resources, e.g., bandwidth, is considerably large. Then, BER is primarily affected by signal attenuation and fading rather than congestion and interference.

\section{ROBOTIC ROUTER OPTIMIZATION CONSIDERING ONLY PATH LOSS}

In this section, we develop the foundation of robotic router optimization, using BER as a metric, by considering only the path-loss component of the channel. This analysis will then serve as a benchmark for our derivations in the subsequent sections, where we take fading into account.

\section{A. Objective Function}

Consider a channel that only experiences path loss. We have the following model for the received SNR in the transmission from the $i$ th robot to the $j$ th one:

$$
\gamma_{i, j}=\frac{\alpha_{i, j}}{d_{i, j}^{n}}
$$

where $d_{i, j}$ is the distance between robots $i$ and $j$, and $\alpha_{i, j}$ is a function of system parameters, such as transmit power, antenna gain, and frequency of operation. Parameter $n$ is the path-loss exponent, which depends on the environment (typically around 2-6) [18].

Without loss of generality, we label the robots as follows: node 1 represents the transmitter, node 2 represents the node that directly receives the information from the transmitter, and so on. Then, we have the following approximated expression, for the probability of correct reception, at the receiving end node:

$$
\begin{aligned}
P_{c}(\mathrm{RX}) & \approx P_{c}(\{m\} \mid\{m-1\}) P_{c}(\{m-1\}) \\
& \approx \prod_{i=2}^{m} P_{c}(\{i\} \mid\{i-1\})
\end{aligned}
$$

where $P_{c}(\{i\})$ represents the probability of correct reception of a bit, at node $i$, and $P_{c}(\{i\} \mid\{i-1\})$ denotes the conditional probability of correct reception of a bit, at the $i$ th node, given correct reception at node $i-1$. By combining (1) and (3), we have the following objective function to maximize: ${ }^{2}$

$$
P_{c}(\mathrm{RX})=\prod_{i=2}^{m}\left(1-0.2 \exp \left\{-c \gamma_{i-1, i}\right\}\right)
$$

\footnotetext{
${ }^{2}$ Note that $P_{c}(\mathrm{RX})=1-P_{b}(\mathrm{RX})$, where $P_{b}(\mathrm{RX})$ is the BER of the receiving node.
} 
where $c=\frac{1.5}{M-1}$. Then, the goal of the routers is to position themselves such that $P_{c}(\mathrm{RX})$ is maximized.

Remark 1: The approximation of (4) is based on only considering correct receptions, i.e., if a bit gets flipped a number of times but is correctly received at the end, we do not consider such a case as a correct reception. As a result, the approximation of (4) becomes a lower bound on $P_{c}(\mathrm{RX})$. The approximation can also be justified by considering the fact that the probability of one bit flip is typically low (less than $10^{-3}$ ). As a result, the probability of more than one flip is typically negligible, which also justifies the approximation of (4).

Remark 2: Note that as $d_{i-1, i} \rightarrow 0, \quad 1-0.2 \exp$ $\left\{-c \gamma_{i-1, i}\right\} \rightarrow 1$, and $\frac{\partial\left(1-0.2 \exp \left\{-c \gamma_{i-1, i}\right\}\right)}{\partial d_{i-1, i}} \rightarrow 0$. Then, $P_{c}(\mathrm{RX})$ is continuous and differentiable in its domain.

\section{B. Optimum Configuration of Robotic Routers}

Let $x_{i} \in \mathbb{R}^{2}$ denote the position of robot $i$. Then, $x=$ $\left[\begin{array}{llll}x_{1}^{\mathrm{T}} & x_{2}^{\mathrm{T}} & \cdots & x_{m}^{\mathrm{T}}\end{array}\right]^{\mathrm{T}}$, and $x_{r}=\left[\begin{array}{llll}x_{2}^{\mathrm{T}} & x_{3}^{\mathrm{T}} & \cdots & x_{m-1}^{\mathrm{T}}\end{array}\right]^{\mathrm{T}}$. We assume that all the robotic routers are the first-order systems: $\dot{x}_{i}=$ $u_{i}$, for $i \in\{2, \ldots, m-1\}$. Let $\mathcal{W} \subset \mathbb{R}^{2}$ denote the valid workspace of the robots. Then, we have the following optimization problem (over $x_{r}$ ), by considering the objective function of (4):

$$
\begin{array}{ll}
\operatorname{maximize} & \mathcal{J}\left(x_{r}\right)=\sum_{i=2}^{m} \ln \left(1-0.2 \exp \left\{-c \gamma_{i-1, i}\right\}\right) \\
\text { subject to } & x_{i} \in \mathcal{W}, \quad \forall i \in\{2, \ldots, m-1\}
\end{array}
$$

where $\quad \gamma_{i-1, i}=\frac{\alpha_{i-1, i}}{d_{i-1, i}^{n}}, \quad d_{i-1, i}=\left\|x_{i-1}-x_{i}\right\|, \quad$ for $\quad i \in$ $\{2, \ldots, m\}$.

Proposition 1: If $n+1 \leq \min _{i}\left\{\frac{n c \gamma_{i-1, i}}{1-0.2 \exp \left\{-c \gamma_{i-1, i}\right\}}\right\}$ all the time, then the optimization problem of (5) is concave for a convex $\mathcal{W}$.

Proof: Let $\mathcal{J}_{i}\left(x_{r}\right)=\ln \left(1-0.2 \exp \left\{-c \gamma_{i-1, i}\right\}\right) \quad$ and $v_{i-1, i}=\frac{\partial \gamma_{i-1, i}}{\partial d_{i-1, i}} \nabla_{x_{i}} d_{i-1, i}$. Then, for $\forall i \in\{2, \ldots, m-1\}$ :

$$
\begin{aligned}
\nabla_{x_{i}}^{2} \mathcal{J}_{i}= & \left(\frac{\partial^{2} \mathcal{J}_{i}}{\partial \gamma_{i-1, i}^{2}}+\frac{\partial \mathcal{J}_{i}}{\partial \gamma_{i-1, i}} \frac{n+1}{n \gamma_{i-1, i}}\right) v_{i-1, i} v_{i-1, i}^{\mathrm{T}} \\
& +\frac{\partial \mathcal{J}_{i}}{\partial \gamma_{i-1, i}} \frac{1}{n \gamma_{i-1, i}}\left(v_{i-1, i} v_{i-1, i}^{\mathrm{T}}-\left\|v_{i-1, i}\right\|^{2} I_{2}\right) \\
\preceq & \left(\frac{\partial^{2} \mathcal{J}_{i}}{\partial \gamma_{i-1, i}^{2}}+\frac{\partial \mathcal{J}_{i}}{\partial \gamma_{i-1, i}} \frac{n+1}{n \gamma_{i-1, i}}\right) v_{i-1, i} v_{i-1, i}^{\mathrm{T}} .
\end{aligned}
$$

Furthermore, it can be shown that $\nabla_{x_{i-1}}^{2} \mathcal{J}_{i}=\nabla_{x_{i}}^{2} \mathcal{J}_{i}=$ $-\nabla_{x_{i-1}} \nabla_{x_{i}} \mathcal{J}_{i}$ for $\forall i \in\{3, \ldots, m-1\}$. Let $\phi_{i}=\nabla_{x_{i}}^{2} \mathcal{J}_{i}$ and $\phi_{m-1}^{\prime}=\nabla_{x_{m-1}}^{2} \mathcal{J}_{m}$. We have $\nabla_{x_{i}}^{2} \mathcal{J}=\sum_{j=i}^{i+1} \phi_{j}$ if $i \in$ $\{2, \ldots, m-2\}$, and $\nabla_{x_{i}}^{2} \mathcal{J}=\phi_{m-1}+\phi_{m-1}^{\prime}$ if $i=m-1$, which results in the following Hessian matrix $H=\nabla_{x_{r}}^{2} \mathcal{J}$ :

$$
H=\left[\begin{array}{ccccc}
\sum_{j=2}^{3} \phi_{j} & -\phi_{3} & 0 & \cdots & 0 \\
-\phi_{3} & \sum_{j=3}^{4} \phi_{j} & -\phi_{4} & \cdots & 0 \\
\vdots & \vdots & \vdots & \ddots & \vdots \\
0 & 0 & 0 & -\phi_{m-1} & \phi_{m-1}+\underbrace{\phi_{m-1}^{\prime}}_{=\phi_{m}}
\end{array}\right] .
$$

We can then write $H$ as a sum of matrices $H_{i}$ such that the nonzero blocks of each $H_{i}$ are only related to $\phi_{i}$. It is then easy to show that a sufficient condition to make (7) negative semidefinite is to force all $\phi_{i}$ s to be negative semidefinite for $i \in\{2, \ldots, m\}$. From (6), this means that $\frac{\partial^{2} \mathcal{J}_{i}}{\partial \gamma_{i-1, i}^{2}}+\frac{\partial \mathcal{J}_{i}}{\partial \gamma_{i-1, i}} \frac{n+1}{n \gamma_{i-1, i}} \leq 0$ for all $i$, or $n+1 \leq \min _{i}\left\{\frac{n c \gamma_{i-1, i}}{1-0.2 \exp \left\{-c \gamma_{i-1, i}\right\}}\right\}$.

Remark 3: One way to ensure the condition of Proposition 1 is to enforce $\min _{i}\left\{\gamma_{i-1, i}\right\} \geq \frac{n+1}{n c}=\frac{(M-1)(n+1)}{1.5 n}$, which is a stronger condition. This condition implies that all the robots need to maintain a minimum received SNR. This requirement increases as the modulation constellation size $M$ increases. However, since $M$ is usually around $4-8$, it should be easy to satisfy this condition most of the time. For instance, for real channel measurements of Fig. 9 in our basement, the estimated path-loss exponent is $\hat{n}=2.32$. Then, the sufficient condition of Proposition 1 is satisfied if $\min _{i}\left\{\gamma_{i-1, i}\right\} \geq 4.57 \mathrm{~dB}$ for $M=4$.

We then propose the following control law:

$$
\begin{aligned}
u_{i} & =\kappa \nabla_{x_{i}} \mathcal{J}\left(x_{r}\right) \\
& =\kappa\left(\frac{\partial \mathcal{J}_{i}\left(x_{r}\right)}{\partial d_{i-1, i}} \frac{x_{i}-x_{i-1}}{d_{i-1, i}}+\frac{\partial \mathcal{J}_{i+1}\left(x_{r}\right)}{\partial d_{i, i+1}} \frac{x_{i}-x_{i+1}}{d_{i, i+1}}\right)
\end{aligned}
$$

where $\kappa$ is a positive constant, and $\mathcal{J}_{i}$ is as defined in Proposition 1. Then, the system will converge to the optimum configuration asymptotically, under the condition of Proposition 1. Since the control input $u_{i}$ only depends on the information of node $i$ and the nodes in its vicinity, control of motion can be implemented in a decentralized way.

Next, we characterize some of the properties of the optimum solution, for the optimization problem of (5).

Proposition 2: In the absence of obstacles, the global optimum of (5) is achieved when all the routers stand on the line segment between $x_{1}$ and $x_{m}$.

Proof: Assume that the robotic routers have reached the optimal configuration, but they are not on the line between the transmitter and the receiver. It can be easily seen that by projecting all the $x_{i}$ s to the line that passes through $x_{1}$ and $x_{m}$, the transmission distances will get smaller, resulting in a higher $P_{c}(\mathrm{RX})$. If any projection falls out of the line segment between $x_{1}$ and $x_{m}$, there is always a position on the line segment that results in a lower BER. Therefore, the global optimum can only be achieved when all the routers stand on the line segment between $x_{1}$ and $x_{m}$. 
Remark 4: Note that Proposition 2 holds for any objective function that is a decreasing function of $d_{i-1, i}$ for $i \in$ $\{2, \ldots, m\}$.

Based on Proposition 2, we have the following simplified optimization problem, in the absence of obstacles:

$$
\begin{array}{ll}
\operatorname{maximize} & \mathcal{J}(d)=\sum_{i=2}^{m} \ln \left(1-0.2 \exp \left\{-c \gamma_{i-1, i}\right\}\right) \\
\text { subject to } & d_{i-1, i} \geq 0, \forall i \in\{2, \ldots, m\}, \mathbf{1}_{m-1}^{\mathrm{T}} d=D
\end{array}
$$

where $d=\left[\begin{array}{llll}d_{1,2} & d_{2,3} & \ldots & d_{m-1, m}\end{array}\right]^{\mathrm{T}}, \mathbf{1}_{m-1}$ is an $(m-1)$ dimensional vector with all entries equal to 1 , and $D=\| x_{1}-$ $x_{m} \|$.

Proposition 3: Assume that the concavity condition of Proposition 1 holds. Then, the optimal solution of (9) satisfies the following properties.

1) If $\alpha_{i-1, i}>\alpha_{j-1, j}$, then $d_{i-1, i}^{\star}>d_{j-1, j}^{\star}$;

2) If $\alpha_{i-1, i}=\alpha_{j-1, j}$, then $d_{i-1, i}^{\star}=d_{j-1, j}^{\star}$;

where $d_{i-1, i}^{\star}$ is the optimum distance between nodes $i-1$ and $i$.

Proof: Clearly, the optimization problem of (9) is concave if the condition in Proposition 1 holds. Consider the dual function of the primal problem of (9): $g(d, \lambda, \nu)=\sum_{i=2}^{m} \mathcal{J}_{i}(d)-$ $\sum_{i=2}^{m} \lambda_{i-1, i} d_{i-1, i}+\nu\left(\mathbf{1}_{m-1}^{\mathrm{T}} d-D\right)$, where $\lambda_{i-1, i}$ and $\nu$ are the Lagrange multipliers. For a concave optimization problem, the optimal primal and dual solutions satisfy the following KarushKuhn-Tucker (KKT) conditions [22]: $\frac{\partial \mathcal{J}_{i}\left(d^{\star}\right)}{\partial d_{i-1, i}^{\star}}-\lambda_{i-1, i}^{\star}+\nu^{\star}=$ $0 ; \lambda_{i-1, i}^{\star} d_{i-1, i}^{\star}=0 ; d_{i-1, i}^{\star} \geq 0 ; \lambda_{i-1, i}^{\star} \geq 0 ; \mathbf{1}_{m-1}^{\mathrm{T}} d^{\star}-D=0$, where $d_{i-1, i}^{\star}, \lambda_{i-1, i}^{\star}$, and $\nu^{\star}$ are the optimal points. This results in the following required condition:

$$
\frac{\partial \mathcal{J}_{i}\left(d^{\star}\right)}{\partial d_{i-1, i}^{\star}}=\frac{\partial \mathcal{J}_{j}\left(d^{\star}\right)}{\partial d_{j-1, j}^{\star}} .
$$

Because of the concavity of the objective function, $\frac{\partial \mathcal{J}_{i}(d)}{\partial d_{i-1, i}}$ is strictly decreasing with respect to $d_{i-1, i}$. It is also straightforward to show that $\frac{\partial \mathcal{J}_{i}(d)}{\partial d_{i-1, i}}$ is strictly increasing with respect to $\alpha_{i-1, i}$. Therefore, the required condition of (10) can only be satisfied when $d_{i-1, i}^{\star}>d_{j-1, j}^{\star}$ if $\alpha_{i-1, i}>\alpha_{j-1, j}$. Similarly, if $\alpha_{i-1, i}=\alpha_{j-1, j},(10)$ holds if and only if $d_{i-1, i}^{\star}=d_{j-1, j}^{\star}$, which completes the proof.

Proposition 3 indicates that the routers should be equally spaced between the transmitter and the receiver if the transceivers of all the robots are homogenous and all the links have the same underlying path-loss parameters. ${ }^{3}$ However, if a link experiences a lower $\alpha_{i-1, i}$ (for instance, due to a lower transmit power), then the corresponding nodes of that link should get closer to each other in order to achieve a better overall performance.

\footnotetext{
${ }^{3}$ The second condition has to do with the size of the space of operation and its environmental features. For instance, if the operation is over a larger space, then the probability to have the same path-loss parameters, over the whole space, is lower.
}

\section{Motion Planning for the Optimization of Robotic Routers}

In the previous section, we characterized the optimum configuration of the routers for different scenarios, by mainly assuming a convex workspace (or no environmental constraints in some cases). In this part, we extend our analysis and consider motion planning to achieve the optimum configuration, in the presence of obstacles. We extend the control law of (8), in order to include obstacle avoidance, using a similar approach to Stump et al. [6]. In this approach, the robotic routers are assumed to operate in a walled environment, which will allow us to add the obstacle avoidance as linear constraints. Let $N_{\perp}$ represent the outward normal vector of one side of a walled obstacle. Then, the $i$ th router will avoid colliding with that side of the obstacle by enforcing the following:

$$
\left\langle N_{\perp}, \dot{x}_{i}\right\rangle=\left\langle N_{\perp}, u_{i}\right\rangle \geq 0 .
$$

We will then have the following optimization problem, by considering the objective function of the previous section, obstacles, and control of motion:

$$
\operatorname{maximize}\left\langle\nabla_{x_{r}} \mathcal{J}, u\right\rangle \quad \text { subject to } W u \geq 0
$$

where $W$ is the collection of all the constraints, similar to (11), caused by all the obstacles. Hence, at $x_{r}$, the routers can first compute $\nabla_{x_{r}} \mathcal{J}$ and $W$ and then find $u$ by solving (12). In the case of a mobile transmitter or receiver, their dynamics can also be included. The optimization framework of (12) basically tries to guide the robots in the direction of the gradient as much as possible, given the constraints of the environment. It can also be implemented in a decentralized manner since each node only needs the information of the nodes in its vicinity. Note that any obstacle can be approximated by a walled obstacle. However, the computational complexity can go up, depending on the shape of the original obstacle and the required approximation accuracy.

We next compare the performance of our proposed BER approach with those of graph-theoretic approaches. In order to have a compatible setup, we need to translate our SNR model to a link weight between 0 and 1 (which is a common approach in the graph-theoretic literature). Consider the following cutoff version of our SNR model:

$$
\gamma_{i, j}\left(d_{i, j}\right)= \begin{cases}\frac{\alpha_{i, j}}{r^{n}}, & \text { if } d_{i, j}<r \\ 0, & \text { if } d_{i, j}>R \\ \frac{\alpha_{i, j}}{d_{i, j}^{n}}, & \text { otherwise }\end{cases}
$$

where $r$ is the saturation distance, $R$ is the cutoff distance, and $n$ is as defined previously. We can then translate this model to link weights as follows:

$$
w_{i, j}\left(d_{i, j}\right)= \begin{cases}1, & \text { if } d_{i, j}<r \\ 0, & \text { if } d_{i, j}>R \\ \left(\frac{r}{d_{i, j}}\right)^{n}, & \text { otherwise. }\end{cases}
$$

Consider the case where both the transmitting and receiving nodes are stationary. We next compare the performance of our proposed BER approach with that of the graphtheoretic approach of [6], in which a measure of the full graph 


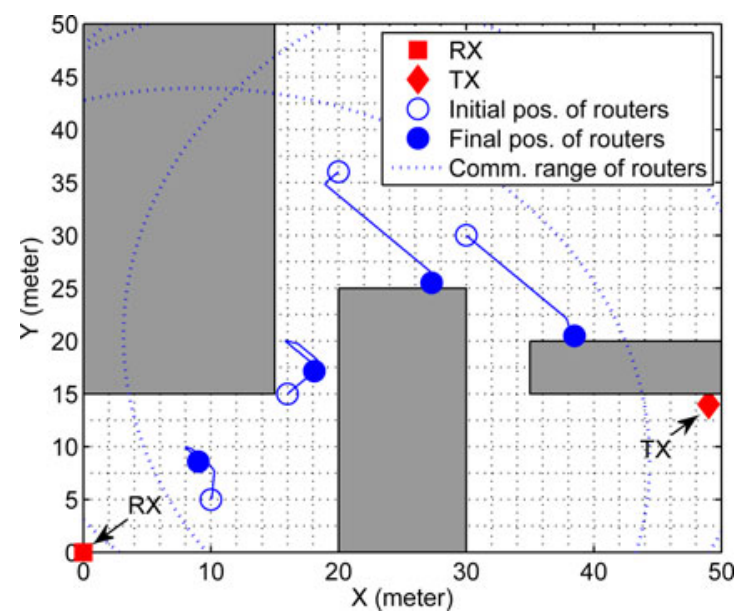

Fig. 1. Robotic router optimization through minimizing the BER (our proposed approach)—gray areas show the obstacles.

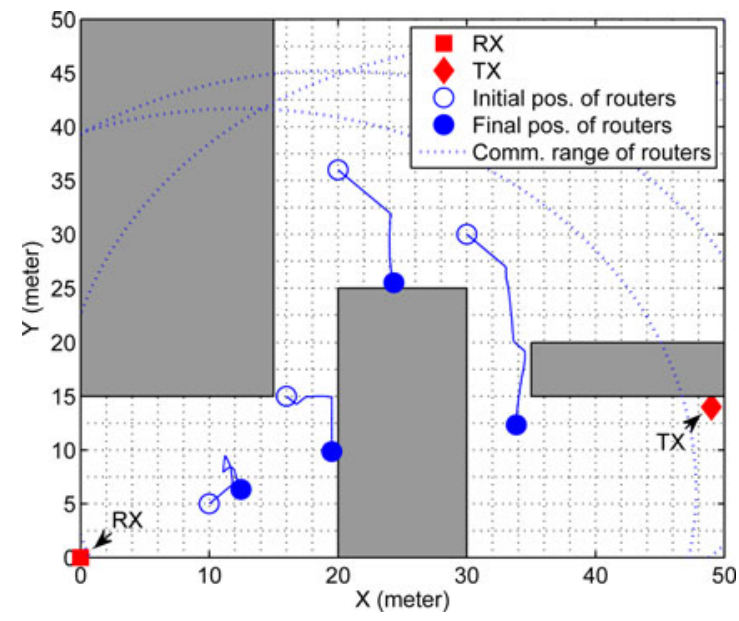

Fig. 2. Robotic router optimization through maximizing the Fiedler eigenvalue [6] - gray areas show the obstacles.

connectivity, the Fiedler eigenvalue, is maximized (similar comparisons should hold with other eigenvalue-based approaches). The following parameters are used: $r=1 \mathrm{~m}, \alpha_{i, j}=2500$, and $R=35.4 \mathrm{~m}$.

Figs. 1 and 2 show the trajectories of four robots, for the cases of minimizing the BER and maximizing the Fiedler eigenvalue, respectively. It can be seen that the final robotic router configurations are not the same. To see the end-to-end performance, Fig. 3 shows the end-to-end BER of the two approaches. Multihop topology is implemented in both cases. It can be seen that our proposed approach performs considerably better (an order of magnitude) and results in a much smaller BER. The figure further confirms that only considering graph-theoretic metrics may not be suitable for the optimization of robotic routers and that communication-oriented metrics of the end-to-end performance, such as BER, should also be considered.

\section{ROBOTIC ROUTER OPTIMIZATION IN FADING ENVIRONMENTS}

In this section, we consider robotic router optimization in fading environments. We start in Section IV-A by presenting the

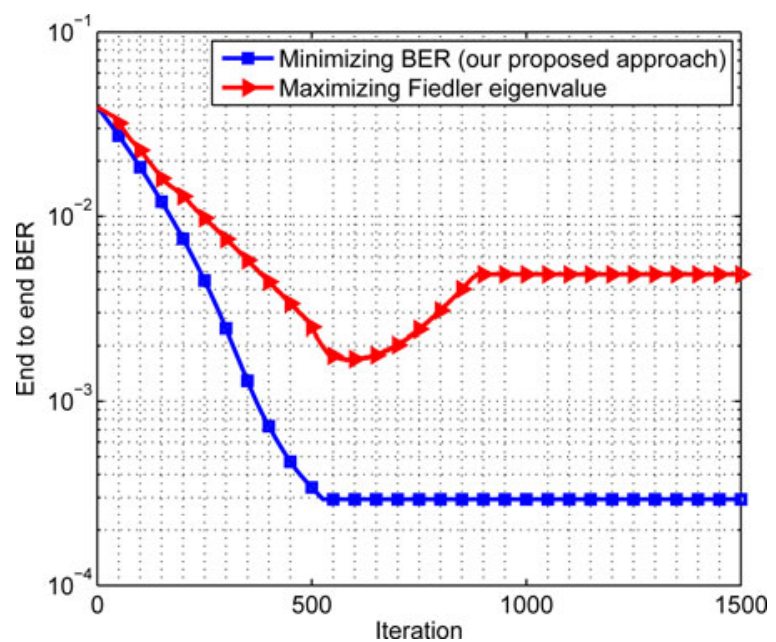

Fig. 3. Comparison of our proposed approach with the case where the Fiedler eigenvalue is maximized. It can be seen that our proposed approach performs considerably better.

general wireless channel model and reviewing the recently developed probabilistic channel prediction framework of Mostofi et al. [16], [17]. In Section IV-B, we develop our motionplanning strategies. We start with the most general framework and then derive a few suboptimum special cases. Section IV-C then shows the performance of the proposed framework.

\section{A. Probabilistic Modeling of a Wireless Channel}

In the wireless communication literature, fading dynamics (and therefore all $\gamma_{i, j} \mathrm{~s}$ ) are characterized probabilistically [18], [23]. By using a 2-D nonstationary random field model, we have the following characterization for $\gamma_{\mathrm{dB}, i, j}=\gamma_{\mathrm{dB}}\left(x_{i}, x_{j}\right)$, the SNR from the node $i$ to the node $j$ (in $\mathrm{dB}$ ):

$$
\begin{aligned}
\gamma_{\mathrm{dB}, i, j}=\gamma_{\mathrm{dB}}\left(x_{i}, x_{j}\right)= & \alpha_{\mathrm{dB}, i, j}-10 n \log _{10}\left(\left\|x_{i}-x_{j}\right\|\right) \\
& +\gamma_{\mathrm{SH}}\left(x_{i}, x_{j}\right)+\gamma_{\mathrm{MP}}\left(x_{i}, x_{j}\right)
\end{aligned}
$$

where $\gamma_{\mathrm{dB}}\left(x_{i}, x_{j}\right)=10 \log _{10}\left(\gamma\left(x_{i}, x_{j}\right)\right)$, and $\gamma_{\mathrm{SH}}\left(x_{i}, x_{j}\right)$ and $\gamma_{\mathrm{MP}}\left(x_{i}, x_{j}\right)$ are independent random variables, representing the effects of shadow fading and multipath fading in $\mathrm{dB}$, respectively [18]. The distance-dependent path loss has a linear decay in the $\mathrm{dB}$ domain. Then, $\alpha_{\mathrm{dB}, i, j}$ and $-10 n$ represent its offset and slope, respectively. Note that, as compared with the pathloss model in (2), $\gamma_{\mathrm{dB}}\left(x_{i}, x_{j}\right)$ of (15) not only depends on the distance between nodes $i$ and $j$ but on their positions as well, due to the additional shadowing and multipath components.

In the communication community, it is well established that a zero-mean Gaussian distribution, with an exponential spatial correlation, can best characterize the distribution of $\gamma_{\mathrm{SH}}\left(x_{i}, x_{j}\right)$ [18], [24]. As for multipath fading, distributions such as Rayleigh, Rician, Nakagami, and lognormal are shown to match its probability density function (pdf) (in the non- $\mathrm{dB}$ domain), depending on the environment [18], [25]. In this section, we assume a lognormal multipath fading and a resulting Gaussian distribution for $\gamma_{\mathrm{MP}}\left(x_{i}, x_{j}\right)$, for the ease of mathematical derivations. We also take $\gamma_{\mathrm{MP}}\left(x_{i}, x_{j}\right)$ to be spatially uncorrelated, since multipath fading typically decorrelates very 
fast, making adaptation to its changes unfeasible. Thus, our channel prediction framework is based on assessing the pathloss and shadowing components. ${ }^{4}$

1) Probabilistic Wireless Channel Assessment Based on a Small Number of Channel Measurements [16], [17]: As can be seen from (15), the channel (in $\mathrm{dB}$ ) between two routers can be modeled as a Gaussian random variable with a certain mean and variance. The corresponding mean and variance can then be predicted, depending on the utilized strategy. For instance, if we only estimate the path-loss term, this term will serve as the mean, with the variance of zero. We next briefly describe how each robot can predict the spatial variations of the instantaneous received SNR, based on the aforementioned model, and by using a small number of available SNR measurements. See [16] and [17] for more details. For the ease of mathematical analysis, we discuss our most general framework, in the context of predicting the link between a fixed node and a moving one at unvisited locations. However, everything is readily applicable to the case of a moving transmitter/receiver [24]. Let $x_{b}$ denote the position of the fixed node. We can write (15) as follows, for the reception quality at position $x: \gamma_{\mathrm{dB}}(x)=$ $\alpha_{\mathrm{dB}}-10 n \log _{10}\left(\left\|x-x_{b}\right\|\right)+\gamma_{\mathrm{SH}}(x)+\gamma_{\mathrm{MP}}(x)$. Let $\mathcal{Q}=$ $\left\{q_{1}, \ldots, q_{k}\right\}$, for $k=|\mathcal{Q}|$, denote the set of the positions, corresponding to the small number of SNR measurements available to the robot. The stacked vector of the received SNR measurements (in $\mathrm{dB}$ ) can then be expressed by $Y=G_{q} \theta+$ $\omega_{\mathrm{SH}}+\omega_{\mathrm{MP}}$, where $G_{q}=\left[\mathbf{1}_{k}-F_{q}\right], \quad F_{q}=\left[10 \log _{10}\left(\| q_{1}-\right.\right.$ $\left.\left.x_{b} \|\right) \cdots 10 \log _{10}\left(\left\|q_{k}-x_{b}\right\|\right)\right]^{\mathrm{T}}, \theta=\left[\begin{array}{ll}\alpha_{\mathrm{dB}} & n\end{array}\right]^{\mathrm{T}}, \omega_{\mathrm{SH}}=$ $\left[\gamma_{\mathrm{SH}}\left(q_{1}\right) \cdots \gamma_{\mathrm{SH}}\left(q_{k}\right)\right]^{\mathrm{T}}$, and $\omega_{\mathrm{MP}}=\left[\gamma_{\mathrm{MP}}\left(q_{1}\right) \cdots \gamma_{\mathrm{MP}}\left(q_{k}\right)\right]^{\mathrm{T}}$.

Based on the commonly used lognormal distribution for shadow fading and its reported exponential spatial correlation, $\omega_{\mathrm{SH}}$ is a zero-mean Gaussian random vector with the covariance matrix $\Omega \in \mathbb{R}^{k \times k}$, where $[\Omega]_{i, j}=\xi_{\mathrm{dB}}^{2} \exp \left\{-\frac{\left\|q_{i}-q_{j}\right\|}{\eta}\right\}$ for $i, j \in\{1, \ldots, k\}$, with $\xi_{\mathrm{dB}}^{2}$ and $\eta$ denoting the variance of the shadow fading component in $\mathrm{dB}$ and its decorrelation distance, respectively. We, furthermore, take $\omega_{\mathrm{MP}}$ to be a zero-mean Gaussian random vector, with the covariance $\rho_{\mathrm{dB}}^{2} I_{k}$, based on the uncorrelated lognormal distribution for multipath fading, where $\rho_{\mathrm{dB}}^{2}$ is the power of multipath fading component (in $\mathrm{dB}$ ). See [17] for more details. We then have the next two lemmas to estimate the underlying model parameters and the channel.

Lemma 1: The least-squares (LS) estimation of the channel parameters is given as follows:

$$
\begin{aligned}
\hat{\theta} & =\left(G_{q}^{\mathrm{T}} G_{q}\right)^{-1} G_{q}^{\mathrm{T}} Y \\
\hat{\xi}_{\mathrm{dB}}^{2}, \hat{\eta} & =\min _{\xi_{\mathrm{dB}}^{2}, \eta} \sum_{l \in \mathcal{L}(l)} \zeta(l)\left[\xi_{\mathrm{dB}}^{2} \exp \{-l / \eta\}-\hat{A}(l)\right]^{2} \\
\hat{\rho}_{\mathrm{dB}}^{2} & =\frac{1}{k} Y_{G_{q}}^{\mathrm{T}} Y_{G_{q}}-\hat{\xi}_{\mathrm{dB}}^{2}
\end{aligned}
$$

where $Y_{G_{q}}=\left(I_{k}-G_{q}\left(G_{q}^{\mathrm{T}} G_{q}\right)^{-1} G_{q}^{\mathrm{T}}\right) Y$ represents the centered version of the measurement vector, and $\hat{A}(l)=$

\footnotetext{
${ }^{4}$ Note that Rayleigh, Rician, and Nakagami provide a better fit than lognormal, for multipath fading, in general. Our oversimplification, however, is only for modeling and analysis. When we show the performance of our framework, the multipath fading component has its natural distribution/correlation.
}
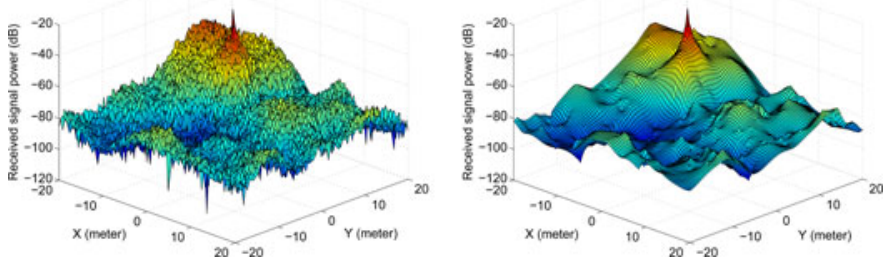

Fig. 4. (Left) Channel generated by our probabilistic simulator with $n=$ $2, \xi_{\mathrm{dB}}=8, \eta=10 \mathrm{~m}$, and $K_{\mathrm{ric}}=5$. (Right) Performance of our channel prediction framework - estimation of the channel (left), with only $5 \%$ channel samples. As can be seen, the estimation captures the main features of the original channel.

$\left(\sum_{(i, j) \in \mathcal{S}(l)}\left[Y_{G_{q}}\right]_{i}\left[Y_{G_{q}}\right]_{j}\right) /|\mathcal{S}(l)|$ is the numerical estimate of the spatial correlation at distance $l$, with $\mathcal{S}(l)=\left\{(i, j) \mid q_{i}, q_{j} \in\right.$ $\left.\mathcal{Q},\left\|q_{i}-q_{j}\right\|=l\right\}$. Furthermore, $\zeta(l)$ is an associated weight that can be chosen, based on the assessment of the accuracy of the estimation of $\hat{A}(l)$, and $\mathcal{L}(l)=\{l \mid 0<\hat{A}(l)<$ $\left.\hat{\xi}_{\mathrm{dB}}^{2}+\hat{\rho}_{\mathrm{dB}}^{2}\right\}$.

Proof: See [16] and [17] for the proof.

Lemma 2: A Gaussian distribution with the following mean, $\Upsilon_{\mathrm{dB}, \mathrm{PL} / \mathrm{SH}}(x)$, and variance, $\sigma_{\mathrm{dB}, \mathrm{PL} / \mathrm{SH}}^{2}(x)$, can predict the distribution of the channel, at an unvisited location $x \in \mathcal{W} \backslash \mathcal{Q}$ :

$$
\begin{aligned}
& \Upsilon_{\mathrm{dB}, \mathrm{PL} / \mathrm{SH}}(x)=\underbrace{G_{x} \hat{\theta}}_{\text {estimated path loss }}+\underbrace{\Psi_{x}^{\mathrm{T}} \Phi^{-1}\left(Y-G_{q} \hat{\theta}\right)}_{\text {estimated shadowing }} \\
& \sigma_{\mathrm{dB}, \mathrm{PL} / \mathrm{SH}}^{2}(x)=\underbrace{\hat{\xi}_{\mathrm{dB}}^{2}+\hat{\rho}_{\mathrm{dB}}^{2}}_{\begin{array}{c}
\text { predictionvar } \\
\text { assuming only path loss }
\end{array}}-\underbrace{\Psi_{x}^{\mathrm{T}} \Phi^{-1} \Psi_{x}}_{\begin{array}{c}
\text { reduction in varwhen } \\
\text { estimating shadowing correlation }
\end{array}}
\end{aligned}
$$

where $\quad G_{x}=\left[1-10 \log _{10}\left(\left\|x-x_{b}\right\|\right)\right], \quad \Phi=\hat{\Omega}+\hat{\rho}_{\mathrm{dB}}^{2} I_{k}$, $[\hat{\Omega}]_{i, j}=\hat{\xi}_{\mathrm{dB}}^{2} \exp \left\{\frac{\left\|q_{i}-q_{j}\right\|}{\hat{\eta}}\right\}, \quad$ and $\Psi_{x}=\left[\hat{\xi}_{\mathrm{dB}}^{2} \exp \left\{-\frac{\left\|x-q_{1}\right\|}{\hat{\eta}}\right\}\right.$ $\left.\cdots \hat{\xi}_{\mathrm{dB}}^{2} \exp \left\{-\frac{\left\|x-q_{k}\right\|}{\hat{\eta}}\right\}\right]^{\mathrm{T}}$. We refer to this predictor as a Probabilistic Path-Loss/Shadowing Estimator in this paper.

Proof: See [16] and [17] for the proof.

To see the performance of this channel predictor, Fig. 4 shows a simulated channel (left) and its prediction by our framework (right), respectively. The simulated channel is generated by our probabilistic channel simulator [23] with $n=2, \xi_{\mathrm{dB}}=8$, and $\eta=10 \mathrm{~m}$. Multipath fading is modeled as an uncorrelated Rician fading variable with parameter $K_{\text {ric }}=5$ [18]. The estimated channel [see the right side of Fig. 4], on the other hand, is reconstructed by using only $5 \%$ random channel measurements in this environment. ${ }^{5}$ It can be seen that the estimated channel captures the path-loss and shadowing components considerably well. However, it does not attempt to predict the multipath fading component (rapid variations) since it is spatially uncorrelated.

Special Case-Probabilistic Path-Loss Estimator: So far, we have established that the SNR of a channel (in $\mathrm{dB}$ ) can be modeled as a Gaussian random variable. Lemma 2 characterizes

\footnotetext{
${ }^{5}$ Note that we use a discretized space to represent the whole channel, i.e., the space consists of a number of very small cells, where we use one value to represent the channel power in each cell. Hence, $5 \%$ random channel measurements mean that the channel power is measured randomly at $5 \%$ of total cells.
} 
the mean and variance of channel prediction if both the path loss and fading correlation/variance are taken into account, as can be seen from (19) and (20). The following more simplified mean and variance, on the other hand, only predict the path-loss component and fading variance, without taking advantage of the shadowing spatial correlation:

$$
\begin{aligned}
& \Upsilon_{\mathrm{dB}, \mathrm{PL}}(x)=G_{x} \hat{\theta} \\
& \sigma_{\mathrm{dB}, \mathrm{PL}}^{2}(x)=\hat{\xi}_{\mathrm{dB}}^{2}+\hat{\rho}_{\mathrm{dB}}^{2} .
\end{aligned}
$$

This estimator is suboptimum and suitable for scenarios where the correlation of shadowing (17) cannot be properly estimated, either due to the lack of enough channel samples or a desire to keep the computational complexity very low.

Remark 5: We use the notation $\Upsilon_{\mathrm{dB}, i, j}=\Upsilon_{\mathrm{dB}}\left(x_{i}, x_{j}\right)$ and $\sigma_{\mathrm{dB}, i, j}=\sigma_{\mathrm{dB}}\left(x_{i}, x_{j}\right)$ to represent a general estimated average SNR (in the dB domain) and its corresponding standard deviation, from node $i$ to $j$. For example, either of the two aforementioned prediction strategies can be used to evaluate these variables. Furthermore, the use of these variables, without the $\mathrm{dB}$, indicates the non- $\mathrm{dB}$ version, e.g., $\Upsilon_{i, j}=10^{\frac{\Upsilon_{\mathrm{dB}, i, j}}{10}}$.

\section{B. Robotic Router Optimization in Fading Environments}

In this part, we propose a framework for robotic router position optimization and motion planning in fading environments, and discuss the underlying tradeoffs. As shown in Section III, our end-to-end performance metric is $P_{c}(\mathrm{RX})=\prod_{i=2}^{m}(1-$ $\left.0.2 \exp \left\{-c 10^{\frac{\gamma_{\mathrm{dB}, i-1, i}}{10}}\right\}\right)$. In this case, however, $\gamma_{\mathrm{dB}, i-1, i}$ is a Gaussian random variable whose mean and variance can be estimated, using either the probabilistic path-loss/shadowing estimator or the probabilistic path-loss estimator. This results in a stochastic $P_{c}(\mathrm{RX})$. A proper approach, common in such cases, is to maximize the average of it, over the predicted distribution of the channels. This results in the following:

$$
\operatorname{maximize} \mathbb{E}\left\{\prod_{i=2}^{m}\left(1-0.2 \exp \left\{-c 10^{\frac{\gamma_{\mathrm{dB}, i-1, i}}{10}}\right\}\right)\right\}
$$

subject to $\quad x_{i} \in \mathcal{W}, \quad \forall i \in\{2, \ldots, m-1\}$

where $\mathbb{E}\{\cdot\}$ denotes the average of the argument. While this optimization problem can be solved numerically, we next show how further simplification is also possible. Based on the aforementioned lognormal distribution for fading, the received SNR, from the $i$ th node to the $j$ th one, is a random variable with the following distribution:

$$
p_{\ln }\left(\gamma_{i, j}\right)=\frac{1}{\sqrt{2 \pi} a \sigma_{\mathrm{dB}, i, j} \gamma_{i, j}} \exp \left\{-\frac{\left(\ln \gamma_{i, j}-a \Upsilon_{\mathrm{dB}, i, j}\right)^{2}}{2\left(a \sigma_{\mathrm{dB}, i, j}\right)^{2}}\right\}
$$

where $a=\frac{\ln 10}{10}$. The first and second moments of $\gamma_{i, j}$ can then be found as $\mathbb{E}\left\{\gamma_{i, j}\right\}=\Upsilon_{i, j} \exp \left\{0.5\left(a \sigma_{\mathrm{dB}, i, j}\right)^{2}\right\}$ and $\mathbb{E}\left\{\gamma_{i, j}^{2}\right\}=$ $\Upsilon_{i, j}^{2} \exp \left\{2\left(a \sigma_{\mathrm{dB}, i, j}\right)^{2}\right\}$, respectively. Following [26], we can approximate a lognormal distribution with a gamma distribution, with the same first and second moments:

$$
p_{\text {gam }}\left(\gamma_{i, j}\right)=\frac{\gamma_{i, j}^{\varphi_{i, j}-1}}{\left(\vartheta_{i, j} \Upsilon_{i, j}\right)^{\varphi_{i, j}} \Gamma\left(\varphi_{i, j}\right)} \exp \left\{-\frac{\gamma_{i, j}}{\vartheta_{i, j} \Upsilon_{i, j}}\right\}
$$

where $\quad \varphi_{i, j}=\left(\exp \left\{\left(a \sigma_{\mathrm{dB}, i, j}\right)^{2}\right\}-1\right)^{-1}, \quad \vartheta_{i, j}=\exp \{1.5$ $\left.\left(a \sigma_{\mathrm{dB}, i, j}\right)^{2}\right\}-\exp \left\{0.5\left(a \sigma_{\mathrm{dB}, i, j}\right)^{2}\right\}$, and $\Gamma(\cdot)$ represents the gamma function. As $\sigma_{\mathrm{dB}, i, j}$ decreases, this approximation becomes better [26]. We then have the following:

$$
\begin{aligned}
& \mathbb{E}\left\{P_{c}(\mathrm{RX})\right\}=\prod_{i=2}^{m}\left(1-0.2 \mathbb{E}\left\{\operatorname { e x p } \left\{-c 10^{\left.\left.\left.\frac{\gamma_{\mathrm{dB}, i-1, i}}{10}\right\}\right\}\right)}\right.\right.\right. \\
& =\prod_{i=2}^{m}\left(1-0.2\left(1+c \vartheta_{i-1, i} 10^{\frac{\Upsilon_{\mathrm{dB}, i-1, i}}{10}}\right)^{-\varphi_{i-1, i}}\right)
\end{aligned}
$$

where the second line can be confirmed, using the distribution of (25), and after a few lines of derivations. Note that in writing (26), we assumed that all the channels are statistically independent, which is an appropriate assumption for several wireless scenarios. We then have the following optimization problem:

$\operatorname{maximize} \sum_{i=2}^{m} \ln \left(1-0.2\left(1+c \vartheta_{i-1, i} 10^{\frac{\Upsilon_{\mathrm{dB}, i-1, i}}{10}}\right)^{-\varphi_{i-1, i}}\right)$

subject to $\quad x_{i} \in \mathcal{W} \quad \forall i \in\{2, \ldots, m-1\}$.

Then, we can use either $\Upsilon_{\mathrm{dB}, \mathrm{PL} / \mathrm{SH}, i-1, i}$ (19) and $\sigma_{\mathrm{dB}, \mathrm{PL} / \mathrm{SH}, i-1, i}(20)$ for $\Upsilon_{\mathrm{dB}, i-1, i}$ and $\sigma_{\mathrm{dB}, i-1, i}$, respectively, or $\Upsilon_{\mathrm{dB}, \mathrm{PL}, i-1, i}(21)$ and $\sigma_{\mathrm{dB}, \mathrm{PL}, i-1, i}$ (22) for $\Upsilon_{\mathrm{dB}, i-1, i}$ and $\sigma_{\mathrm{dB}, i-1, i}$, respectively, depending on our channel prediction strategy. In Propositions 1 and 3, we derived conditions to guarantee the optimality of our robotic router optimization framework, in path-loss environments, and showed the properties of the optimum solution. The Appendix extends those propositions to fading environments, for the probabilistic objective function of (27) and the case of probabilistic path-loss estimator.

1) Suboptimum Deterministic Alternative for Fading Environments: In order to avoid the distribution approximation of (25) as well as the computational complexity, which is associated with the objective function of (27), a simpler alternative is to use the average of the probabilistic channel estimator as an estimate of the channel (in $\mathrm{dB}$ ). Consider the probabilistic estimator of Lemma 2, for instance. The estimated channel average $\Upsilon_{\mathrm{dB}, \mathrm{PL} / \mathrm{SH}}$ is the MMSE estimate of the channel. Thus, we can use this estimation directly in $P_{c}(\mathrm{RX})$. This results in the following suboptimum optimization problem: ${ }^{6}$

$$
\begin{array}{ll}
\operatorname{maximize} & \sum_{i=2}^{m} \ln \left(1-0.2 \exp \left\{-c 10^{\frac{\Upsilon_{\mathrm{dB}, i-1, i}}{10}}\right\}\right) \\
\text { subject to } & x_{i} \in \mathcal{W}, \quad \forall i \in\{2, \ldots, m-1\} .
\end{array}
$$

Then, we can use either $\Upsilon_{\mathrm{dB}, \mathrm{PL} / \mathrm{SH}, i-1, i}$ or $\Upsilon_{\mathrm{dB}, \mathrm{PL}, i-1, i}$, for $\Upsilon_{\mathrm{dB}, i-1, i}$.

Special Case-Deterministic Path-Loss Estimator: In case the average of the probabilistic path-loss estimator is used, i.e., $\Upsilon_{\mathrm{dB}, i-1, i}=\Upsilon_{\mathrm{dB}, \mathrm{PL}, i-1, i}$, the deterministic optimization problem of (28) becomes the same as our optimization problem of the previous section, for the case of path loss only (5). In other words, in this case, the routers model the channel only with the path-loss term, while the channel experiences fading.

\footnotetext{
${ }^{6} \mathrm{It}$ is suboptimum since we are not using the variance information.
} 
2) Summary and Comparison of the Proposed Approaches: We proposed the probabilistic optimization problem of (23), along with the probabilistic channel assessment framework of Lemmas 1 and 2, for robotic router optimization in fading environments. We furthermore showed how this original problem can be simplified to three suboptimum alternatives. The following list summarizes the discussed approaches.

1) Deterministic path-loss estimator (benchmark): In this case, the routers assume that the channel only experiences the distance-dependent path loss, while in a fading environment. Then, the optimization framework of (28) is implemented, where $\Upsilon_{\mathrm{dB}, i-1, i}=\Upsilon_{\mathrm{dB}, \mathrm{PL}, i-1, i}$. This approach is the most compatible with the existing methods in the literature. However, to make it more realistic, instead of assuming a certain decay rate for the wireless signal, the routers estimate the path-loss parameters using (16). This approach becomes the same as the framework of the previous section [see (5)], i.e., Propositions 1-3 hold.

2) Probabilistic path-loss estimator: This refers to the proposed approach of (23) (or 27), where the objective function was averaged over the distribution of fading. However, the spatial correlation of shadowing was not considered when assessing the channel. In this case, the optimization framework of (27) is implemented, where $\Upsilon_{\mathrm{dB}, i-1, i}=$ $\Upsilon_{\mathrm{dB}, \mathrm{PL}, i-1, i}$, and $\sigma_{\mathrm{dB}, i-1, i}=\sigma_{\mathrm{dB}, \mathrm{PL}, i-1, i}$ [from (21) and (22)]. Propositions 5 and 6 then characterize the optimality and properties of the resulting router optimization problem.

3) Deterministic path-loss/shadowing estimator: In this case, the routers predict both the path-loss and shadowing components of the channel, using (19) (which is the conditional average). This conditional average is then treated as the estimate of the channel in (28): $\Upsilon_{\mathrm{dB}, i-1, i}=$ $\Upsilon_{\mathrm{dB}, \mathrm{PL} / \mathrm{SH}, i-1, i}$.

4) Probabilistic path-loss/shadowing estimator: This is the most complete case, in terms of channel assessment and router optimization. Both the objective function and the channel are treated probabilistically, by predicting the path-loss and shadowing components [see (19) and (20)]. The optimization framework of (27) is implemented, with $\Upsilon_{\mathrm{dB}, i-1, i}=\Upsilon_{\mathrm{dB}, \mathrm{PL} / \mathrm{SH}, i-1, i}$ and $\sigma_{\mathrm{dB}, i-1, i}=\sigma_{\mathrm{dB}, \mathrm{PL} / \mathrm{SH}, i-1, i}$.

5) Probabilistic path-loss/shadowing estimator with jitter: This is the same as the previous approach but with one additional step. In order to take advantage of the rapid variations of multipath fading [see the left side of Fig. 4], after the router converges (based on the previous approach), it jitters around a small local area, in order to gather additional measurements on multipath fading, and finds the optimum position which is based on the new samples. We discuss this approach further in the next section.

The aforementioned list is ordered from the most simplified optimization framework to the most complete one. As such, we expect that the performance improves over the list, as verified by the simulation results of the next section. This comes at the cost of an increase in the computation. As the communication modeling gets more complete over the list, it requires more in-

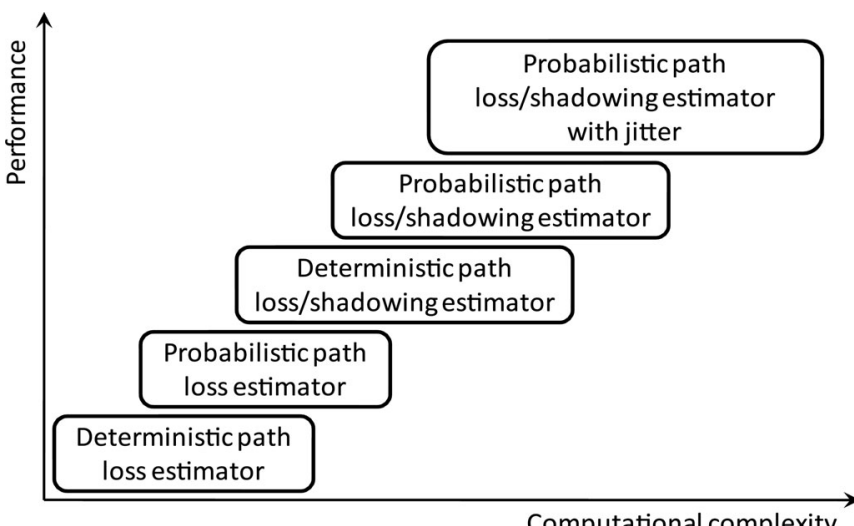

Computational complexity

Fig. 5. Summary of the performance and computational complexity of different approaches.

formation to build the corresponding correlation functions (not that much more though). Consequently, building the correlation functions increases the computation. For instance, if we only know the channel a priori at a couple of locations, then it may be harder to develop the probabilistic path-loss/shadowing estimator, as compared with the probabilistic path-loss case. The routers can also start with a more simplified approach and switch to a better one as they gather more channel samples. Fig. 5 compares the performance and computational complexity of different strategies.

Remark 6: In general, if the optimization framework is only a function of the distances between the routers, then the globally optimum configuration lies on the line segment between the transmitter and the receiver, in the absence of obstacles, as was proved in Proposition 2. As can be seen, this is the case for the first two strategies. However, for the other three cases, this is not the case since we use a more complete channel assessment framework.

\section{Performance of the Proposed Robotic Router Optimization Framework}

In this section, we show the performance of our proposed five strategies of Section IV-B in fading environments. For all the approaches, the performance measure is the average end-to-end BER. From the left side of Fig. 4, it can be seen that a realistic channel and the resulting optimization problem may have many local maxima. Hence, there is no general control law to drive the routers to the global optimum, even with perfect channel knowledge. Therefore, in our framework, each router uses a localized window search approach to solve the optimization problem iteratively, starting from its initial position. More specifically, the router uses the proposed channel estimation strategies, in order to calculate the BER and the overall objective function in a local window around its current position, finds the motion direction that maximizes the objective function by searching the local window, and moves one step in that direction. The performance of this approach depends on the window size and channel estimation quality. If the window size is large enough and the estimation quality is good, then the router is more likely to find the global optimum. 


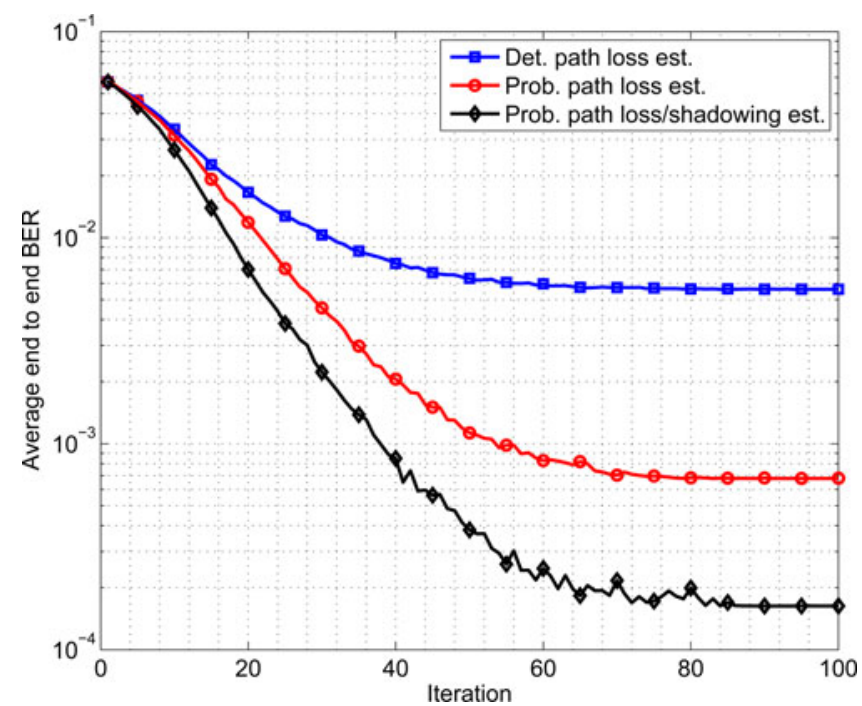

Fig. 6. Performance of the proposed approaches in optimizing a robotic router network in a fading environment - comparison of the deterministic path-loss estimator, probabilistic path-loss estimator, and probabilistic path-loss/shadowing estimator. The channels are generated by our probabilistic channel simulator with $n=2, \xi_{\mathrm{dB}}=10, \eta=10 \mathrm{~m}$, and $K_{\mathrm{ric}}=5$. As can be seen, the proposed probabilistic path-loss estimator and probabilistic path-loss/shadowing estimator perform considerably better.

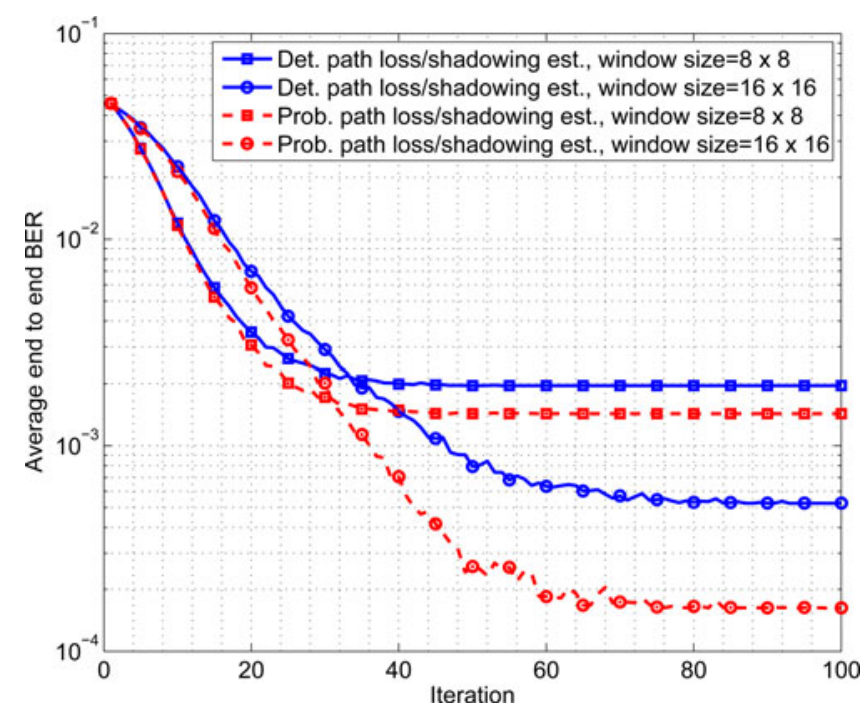

Fig. 7. Performance of the proposed approaches in optimizing a robotic router network in a fading environment-comparison of the deterministic and probabilistic path-loss/shadowing estimators. The channels are generated by our probabilistic channel simulator with $n=2, \xi_{\mathrm{dB}}=8, \eta=10 \mathrm{~m}$, and $K_{\text {ric }}=5$.

First, consider the case of one router, with no obstacles in the environment and a channel that is generated, using our probabilistic channel simulator [23]. Since the performance depends on the initial position of the router and the specific generated channel, we average the performance of each case over several runs of different channel samples (but with the same underlying parameters), as well as random initial router position. Figs. 6-8 show the performance of these approaches. In all the cases, the router uses only $5 \%$ a priori random channel measurements in the environment, in order to estimate the underlying channel

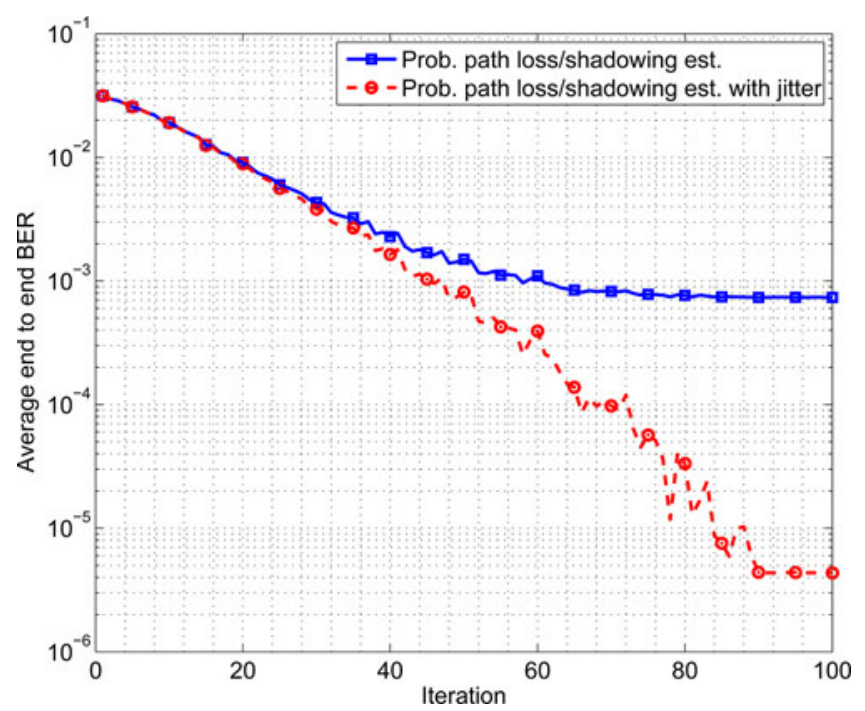

Fig. 8. Impact of adding jitter to mitigate multipath fading. The channels are generated by our probabilistic channel simulator with $n=2, \xi_{\mathrm{dB}}=4$, $\eta=10 \mathrm{~m}$, and $K_{\text {ric }}=2$.

parameters. These measurements could be gathered along the trajectory of the robot (or a number of them cooperatively).

Fig. 6 compares the performance of the deterministic pathloss estimator, probabilistic path-loss estimator, and probabilistic path-loss/shadowing estimator, where a local window search size of $16 \mathrm{~m} \times 16 \mathrm{~m}$ is utilized. The environment is taken to be a square of size $40 \mathrm{~m} \times 40 \mathrm{~m}$ for all the three figures. As can be seen, the deterministic path-loss estimator performs the worst, since it does not account for channel fading. By taking the variance, which is caused by fading, into account, the probabilistic path-loss estimator performs much better (around one order of magnitude). Finally, by considering channel spatial correlation, the probabilistic path-loss/shadowing estimator performs the best.

Next, Fig. 7 compares the performance of the robotic router formation, for the deterministic and probabilistic pathloss/shadowing estimators and two different local window search sizes. As expected, the probabilistic case has a better performance, and the improvement increases as the size of the search window increases.

Finally, Fig. 8 shows the performance with the added jitter strategy. The local window search size is $16 \mathrm{~m} \times 16 \mathrm{~m}$, the environment size is $40 \mathrm{~m} \times 40 \mathrm{~m}$, and the jitter area is $1.6 \mathrm{~m} \times 1.6 \mathrm{~m}$. Since multipath fading is unpredictable, it can severely degrade the estimation performance. Hence, the router can benefit considerably from the proposed jitter strategy as can be seen.

Next, we show the performance of the proposed framework in the presence of obstacles and by using the parameters of a real channel. We also compare the performance with the existing Fiedler eigenvalue approach. For comparison, we consider a cutoff version of our average SNR model, similar to (13), which translates to link weights, as described for (14). We use the pioneer robot of the left side of Fig. 9 to make several measurements in our basement. The right side of Fig. 9 shows a color map of the communication signal strength in the area of interest [23]. Using the proposed approach of Section IV-A, we 


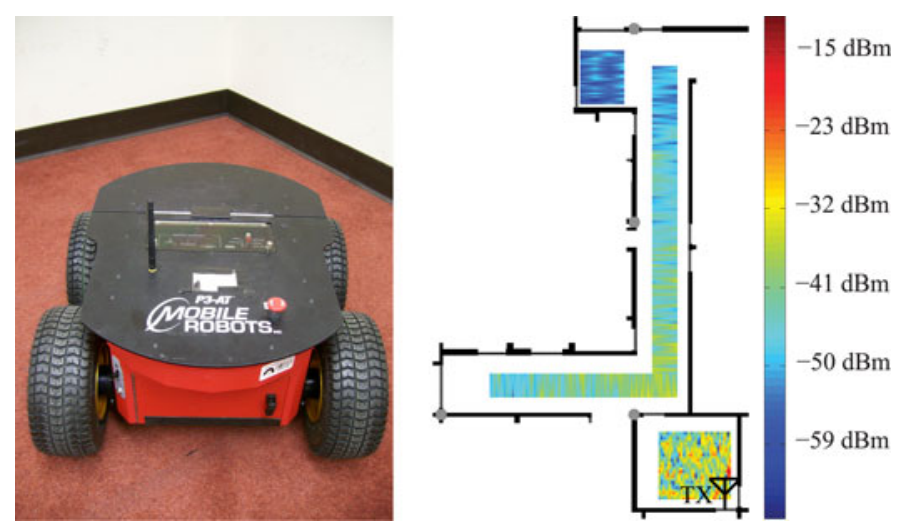

Fig. 9. Channel measurement in our basement. A pioneer robot that gathered the measurements (left) and the measured communication signal strength map to the TX marked on the figure (right).
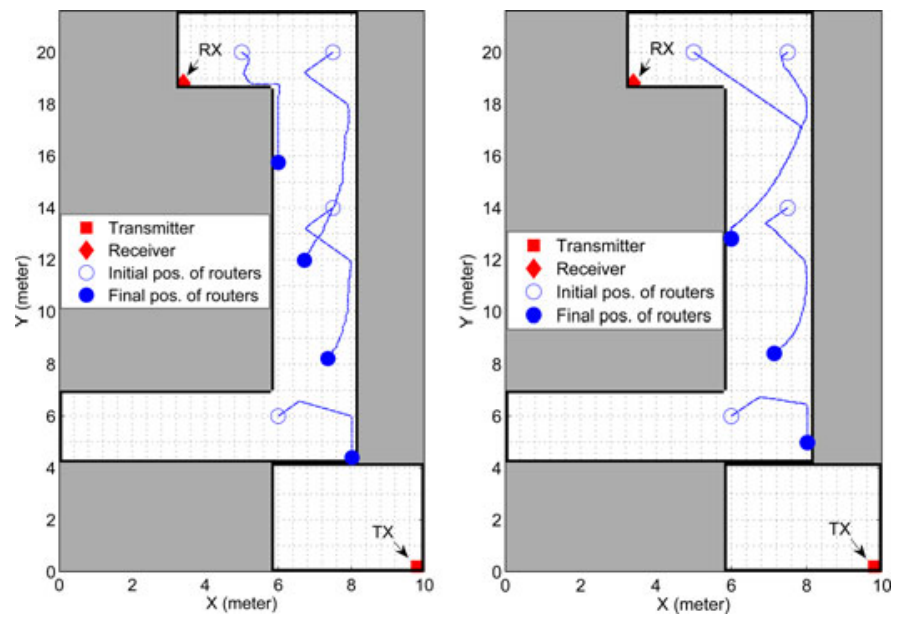

Fig. 10. Robotic router optimization in a fading environment. (Left) Optimum configuration by using probabilistic path-loss estimator (our proposed approach). (Right) Optimum configuration by maximizing the Fiedler eigenvalue [6] - gray areas show the obstacles.

extract the underlying parameters of the measured channel to be as follows: $\hat{\alpha}=3320, \hat{n}=2.32, \hat{\xi}_{\mathrm{dB}}^{2}=11.68, \hat{\eta}=1.20$, and $\hat{\rho}_{\mathrm{dB}}^{2}=9.99$.

Then, we simulate the performance of robotic router formation in an environment with the same underlying channel parameters. Fig. 10 shows the trajectories of four routers, for the case of probabilistic path-loss estimator (left) and maximizing the Fiedler eigenvalue (right). As can be seen, the optimum configurations of the two approaches are different, similar to Figs. 1 and 2. By maximizing the Fiedler eigenvalue, two nodes almost converge to the same position, which is a waste of the resources. Fig. 11 then compares the average BER of the two approaches. Similar to Section III-C, our proposed approach performs considerably better. ${ }^{7}$ In Section VI, we will further

\footnotetext{
${ }^{7}$ On the right side of Fig. 10, two routers almost converge to the same location to maximize the Fiedler eigenvalue because of the environmental constraints. The routers will spread out for both cases, if we use three routers instead. In this case, the average end-to-end BER to maximize the Fiedler eigenvalue is $1.5 \times$ $10^{-3}$, while the average end-to-end BER by using our proposed probabilistic path-loss estimator approach is $3.1 \times 10^{-4}$. It can be seen that our approach still outperforms considerably.
}

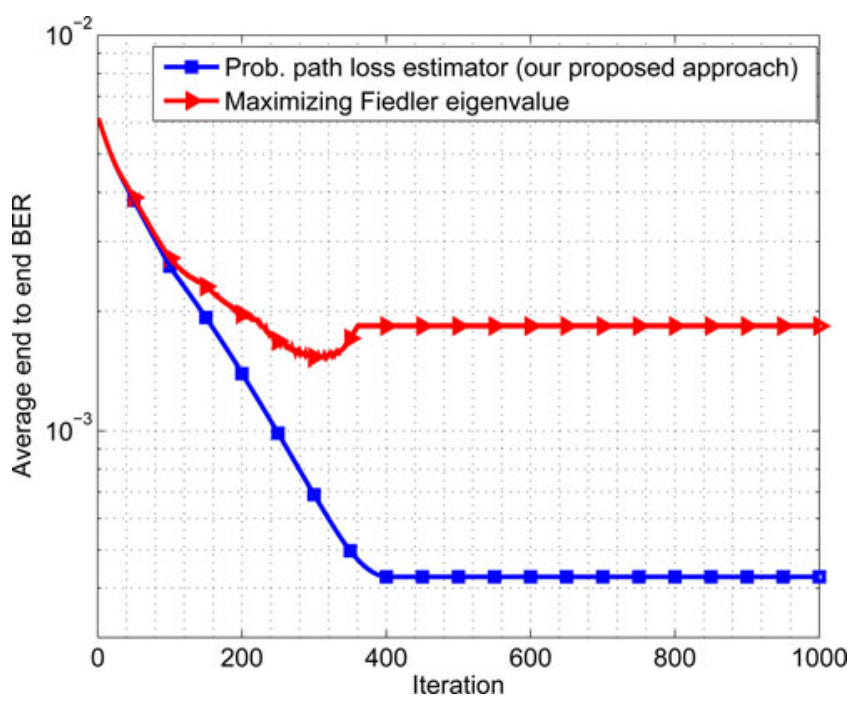

Fig. 11. Comparison of our proposed approach with the case where the Fiedler eigenvalue is maximized in a fading environment. It can be seen that our proposed approach performs considerably better.

verify the effectiveness of our framework by running a preliminary experiment.

\section{COMmunication And Motion Power Management IN ROBOTIC ROUTERS}

In several applications, robotic networks have to work under power constraints. Thus, in this section, we extend our framework and consider the optimization of robotic routers under power constraints. The limited power can be utilized for communication or motion, which results in interesting underlying tradeoffs. The motion and communication costs depend on the scenario. For instance, if the robot is in a bad location in terms of communication, then it may be more cost effective to move, as compared with increasing the transmission power. If that is not the case, the motion can cost more. We will explore such tradeoffs in Section V-C. However, first we begin by understanding the impact of communication power limitations, without considering motion costs, in Sections V-A and B. We then extend our analysis to explore the underlying tradeoffs between motion and communication.

\section{A. Robotic Router Optimization Under Communication Power Constraints Considering Only Path Loss}

In the previous sections, we considered the case where the transmit power of each robot was fixed. In this part, we consider the case where each robot can adapt its transmit power. The goal is then to minimize the overall power consumption of the network while maintaining a minimum required reception quality for all the links. Consider a channel that only experiences path loss. We can express (2) in terms of the given transmit power as follows: $\gamma_{i, j}=\frac{\beta_{i, j} P_{T, i}}{d_{i, j}^{n}}$, where $\beta_{i, j}$ is a function of system parameters, and $P_{T, i}$ is the transmit power of robot $i$. Note that $\alpha_{i, j}=\beta_{i, j} P_{T, i}$. 
Let $P_{b, i-1, i, T}$ and $P_{b, i-1, i}$ represent the maximum tolerable BER and the true BER in the transmission from node $i-1$ to node $i$, respectively. ${ }^{8}$ We require that $P_{b, i-1, i} \leq P_{b, i-1, i, T}$, for all $i \in\{2, \ldots, m\}$, which results in $\gamma_{i-1, i} \geq \gamma_{i-1, i, T}$, where $\gamma_{i-1, i, T}$ is the corresponding SNR threshold: $\gamma_{i-1, i, T}=$ $-\frac{M-1}{1.5} \ln \left(5 P_{b, i-1, i, T}\right)$, using (1). In this part, we are interested in minimizing the overall transmission power while maintaining the minimum required link quality. For the case of no obstacles, it can be easily confirmed that the optimum solution of this problem also lies on the line segment between the transmitter and the receiver. We then have the following optimization problem, considering minimizing the communication power:

$$
\begin{array}{cl}
\operatorname{minimize} & P_{T, \mathrm{RR}}\left(P_{T}, d\right)=\mathbf{1}_{m-1}^{\mathrm{T}} P_{T} \\
\text { subject to } & \gamma_{i-1, i, T} d_{i-1, i}^{n} \leq \beta_{i-1, i} P_{T, i-1}, \quad d_{i-1, i} \geq 0 \\
& \forall i \in\{2, \ldots, m\}, \mathbf{1}_{m-1}^{\mathrm{T}} d=D
\end{array}
$$

where $\quad P_{T}=\left[\begin{array}{llll}P_{T, 1} & P_{T, 2} & \ldots & P_{T, m-1}\end{array}\right]^{\mathrm{T}}, \quad d=\left[\begin{array}{lll}d_{1,2} & d_{2,3} & \ldots\end{array}\right.$ $\left.d_{m-1, m}\right]^{\mathrm{T}}, D=\left\|x_{1}-x_{m}\right\|$, and $P_{T, i}$ is the transmit power of node $i$.

Proposition 4: The solution to the optimization problem of (29) is $d_{i-1, i}^{\star}=\frac{\left(\frac{\beta_{i-1, i}}{\gamma_{i-1, i, T}}\right)^{\frac{1}{n-1}}}{\sum_{j=2}^{m}\left(\frac{\beta_{j-1, j}}{\gamma_{j-1, j, T}}\right)^{\frac{1}{n-1}}} D$, and $P_{T, i-1}^{\star}=$ $\frac{\gamma_{i-1, i, T}}{\beta_{i-1, i}} d_{i-1, i}^{\star n}$, for all $i \in\{2, \ldots, m\}$.

Proof: It can be easily confirmed that the objective function and all the constraints of (29) are convex functions of variables $d$ and $P_{T}$. Consider the dual function of the primal problem: $g\left(P_{T}, d, \lambda, \mu, \nu\right)=\sum_{i=2}^{m} \lambda_{i-1, i}\left(\gamma_{i-1, i, T} d_{i-1, i}^{n}-\beta_{i-1, i}\right.$ $\left.P_{T, i-1}\right)+\mathbf{1}_{m-1}^{\mathrm{T}} P_{T}-\sum_{i=2}^{m} \mu_{i-1, i} d_{i-1, i}+\nu\left(\mathbf{1}_{m-1}^{\mathrm{T}} d-D\right)$, where $\lambda_{i-1, i}, \mu_{i-1, i}$, and $\nu$ are the Lagrange multipliers. Therefore, KKT conditions must be satisfied: $1-\lambda_{i-1, i}^{\star} \beta_{i-1, i}=$ $0 ; \quad n \lambda_{i-1, i}^{\star} d_{i-1, i}^{\star n-1} \gamma_{i-1, i, T}+\nu^{\star}-\mu_{i-1, i}^{\star}=0 ; \quad \lambda_{i-1, i}^{\star}\left(\gamma_{i-1, i, T}\right.$ $\left.d_{i-1, i}^{\star n}-\beta_{i-1, i} P_{T, i-1}^{\star}\right)=0 ; \quad \mu_{i-1, i}^{\star} A_{i-1, i}^{\star}=0 ; \quad \lambda_{i-1, i}^{\star} \geq 0$; $\mu_{i-1, i}^{\star} \geq 0 ; \quad d_{i-1, i}^{\star} \geq 0 ; \quad \beta_{i-1, i} P_{T, i-1}^{\star}-\gamma_{i-1, i, T} d_{i-1, i}^{\star n} \geq 0 ;$ $\mathbf{1}_{m-1}^{\mathrm{T}} d^{\star}-D=0$, where $P_{T, i-1}^{\star}, d_{i-1, i}^{\star}, \lambda_{i-1, i}^{\star}, \mu_{i-1, i}^{\star}$, and $\nu^{\star}$ are the optimal points. Then, we have $\left(\frac{\gamma_{i-1, i, T}}{\beta_{i-1, i}}\right)^{\frac{1}{n-1}} d_{i-1, i}^{\star}=$ $\left(\frac{\gamma_{j-1, j, T}}{\beta_{j-1, j}}\right)^{\frac{1}{n-1}} d_{j-1, j}^{\star}$, which results in the optimum solution of Proposition 4.

If $\frac{\gamma_{i-1, i, T}}{\beta_{i-1, i}}=\frac{\gamma_{j-1, j, T}}{\beta_{j-1, j}}$, for $\forall i, j \in\{2, \ldots, m\}$, all the routers should be equally spaced between the transmitter and the receiver.

The analysis of this section then allows us to mathematically characterize the benefit that we gain by using robotic routers, as compared with increasing the communication power of the transmitting node in order to have a direct transmission. We next formulate this. From the solution of Proposition 4, we know that the total power consumption, for the case of using

\footnotetext{
${ }^{8}$ Note that $P_{b, i-1, i, T}$ should be a function of the total number of nodes, as well as the end-to-end performance requirement, in order to properly utilize the routers.
}

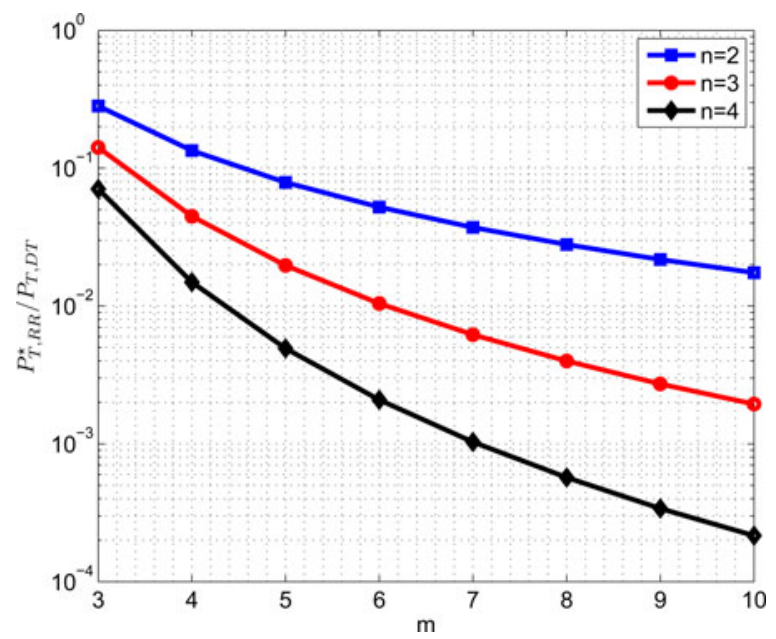

Fig. 12. Comparing the performance of a robotic router network with that of a direct transmission, in terms of total power consumption. The $y$-axis shows the power ratio of (31).

robotic routers, is

$$
P_{T, \mathrm{RR}}^{\star}=\sum_{i=2}^{m} P_{T, i-1}^{\star}=\frac{D^{n}}{\left(\sum_{i=2}^{m}\left(\frac{\beta_{i-1, i}}{\gamma_{i-1, i, T}}\right)^{\frac{1}{n-1}}\right)^{n-1}}
$$

and the total power consumption for direct transmission, without using robotic routers, is $P_{T, \mathrm{DT}}=\frac{\gamma_{1, m, T}}{\beta_{1, m}} D^{n}$, where $\gamma_{1, m, T}$ is the required SNR from the TX node to the RX. Without loss of generality, assume that $\beta_{1, m}=\beta_{i-1, i}=\beta$ and $P_{b, i-1, i, T}=P_{b, T}$ for $\forall i \in\{2,3, \ldots, m\}$. Then, in order to have the same end-to-end performance $\left(P_{b, 1, m, T}\right)$ for both cases, the following target BER can be used for each link, for the case of using robotic routers: $P_{b, T}=1-\left(1-P_{b, 1, m, T}\right)^{\frac{1}{m-1}}$, which results in $\gamma_{1, m, T}=-\frac{M-1}{1.5} \ln \left(5 P_{b, 1, m, T}\right)$ and $\gamma_{i-1, i, T}=$ $-\frac{M-1}{1.5} \ln \left(5\left(1-\left(1-P_{b, 1, m, T}\right)^{\frac{1}{m-1}}\right)\right)$. Then, we have the following power ratio:

$$
\frac{P_{T, \mathrm{RR}}^{\star}}{P_{T, \mathrm{DT}}}=\frac{\ln \left(5\left(1-\left(1-P_{b, 1, m, T}\right)^{\frac{1}{m-1}}\right)\right)}{\ln \left(5 P_{b, 1, m, T}\right)(m-1)^{n-1}} .
$$

From (31), it can be seen that the ratio decreases as $n$ (the exponent of the distance-dependent path loss) increases. This is due to the fact that the signal strength drops faster as $n$ increases. Thus, the direct transmission case has to increase the transmit power considerably. Furthermore, if $m$ (the total number of robots) increases, the ratio decreases, as expected. This result can also be interpreted as follows. If both systems are given equal communication power, the robotic router case will perform considerably better. Fig. 12 shows $\frac{P_{T, \mathrm{RR}}^{\star}}{P_{T, \mathrm{DT}}}$ of (31) with $P_{b, 1, m, T}=10^{-3}$. As can be seen, the robotic router network can reduce communication power consumption considerably as $n$ or $m$ increases.

We conclude this part by noting that the benefits of utilizing robotic routers is beyond just saving on the overall communication power. They also provide robustness through reconfigurability. For instance, if we have mobile TX and RX 
nodes, depending on their positions, the direct link quality can degrade considerably. The router network can then reconfigure properly to ensure reliable flow of information.

\section{B. Robotic Router Optimization Under Communication Power Constraints in Fading Environments}

We next consider the impact of power constraints on robotic router optimization in fading environments. Similar to the previous section, we minimize the total power consumption while requiring each link to maintain a minimum acceptable performance. In this case, the performance is measured by the average BER, as discussed in Section IV-B. Let $\bar{P}_{b, i-1, i, T}$ denote the maximum acceptable average BER for the link between the $(i-1)$ th and $i$ th nodes, i.e., we require that $\bar{P}_{b, i-1, i} \leq \bar{P}_{b, i-1, i, T}$ for all $i \in\{2, \ldots, m\}$. This requirement translates to the following SNR requirement, by using the moment-generating function of a gamma distribution [see the derivations of (26)]:

$$
\Upsilon_{i-1, i} \geq \frac{\left(5 \bar{P}_{b, i-1, i, T}\right)^{-\frac{1}{\varphi_{i-1, i}}}-1}{c \vartheta_{i-1, i}}=\Upsilon_{i-1, i, T}
$$

where $\varphi_{i-1, i}, \vartheta_{i-1, i}$, and $c$ are as defined in the previous sections. Consider the case of probabilistic path-loss estimator in Section IV-B, which estimates the path loss and fading variance. Then, the optimization problem of (29) can be modified as follows:

$$
\begin{array}{ll}
\operatorname{minimize} & P_{T, \mathrm{FD}}\left(P_{T}, d\right)=\mathbf{1}_{m-1}^{\mathrm{T}} P_{T} \\
\text { subject to } & \Upsilon_{\mathrm{PL}, i-1, i, T} d_{i-1, i}^{n} \leq \beta_{i-1, i} P_{T, i-1}, \quad d_{i-1, i} \geq 0 \\
& \forall i \in\{2, \ldots, m\}, \mathbf{1}_{m-1}^{\mathrm{T}} d=D
\end{array}
$$

where $\Upsilon_{\mathrm{PL}, i-1, i, T}=\frac{\left(5 \bar{P}_{b, i-1, i, T}\right)^{-\frac{1}{\overline{P L}, i-1, i_{1}}-1}}{c \vartheta_{\mathrm{PL}, i-1, i}}, \quad \varphi_{\mathrm{PL}, i-1, i}=$ $\left(\exp \left\{\left(a \sigma_{\mathrm{dB}, \mathrm{PL}, i-1, i}\right)^{2}\right\}-1\right)^{-1}, \quad \vartheta_{\mathrm{PL}, i-1, i}=\exp \{1.5$ $\left.\left(a \sigma_{\mathrm{dB}, \mathrm{PL}, i-1, i}\right)^{2}\right\}-\exp \left\{0.5\left(a \sigma_{\mathrm{dB}, \mathrm{PL}, i-1, i}\right)^{2}\right\}$, and $a=\frac{\ln 10}{10}$. Then, Proposition 4 can characterize the solution of (33) by replacing $\gamma_{i-1, i, T}$ with $\Upsilon_{\mathrm{PL}, i-1, i, T}$. Because of fading, however, $\Upsilon_{\mathrm{PL}, i-1, i, T}$ is higher than $\gamma_{i-1, i, T}$ if we take $\bar{P}_{b, i-1, i, T}=$ $P_{b, i-1, i, T}$. It is also easy to confirm that the two solutions become the same if $\sigma_{\mathrm{dB}, \mathrm{PL}, i-1, i} \rightarrow 0$ for all $i$, i.e., fading goes to zero.

We can now mathematically characterize how much we benefit from properly accounting for fading, as opposed to using only a path-loss model (case of deterministic path-loss estimator in Section IV-B), for robotic router optimization in fading environments. Let $P_{T \text {.FD }}^{\star}$ denote the total power consumption of the robotic router, as a result of the optimization of (33). To have a fair comparison, assume that the robotic router system that only considers path loss and does not account for fading, is given the same total power. Then, the best performance of the path-loss-only case is achieved by solving the following optimization problem:

$$
\begin{array}{ll}
\operatorname{maximize} & \gamma_{T} \\
\text { subject to } & \gamma_{T} d_{i-1, i}^{n} \leq \beta_{i-1, i} P_{T, i-1}, \quad d_{i-1, i} \geq 0 \\
& \forall i \in\{2, \ldots, m\}, \quad \mathbf{1}_{m-1}^{\mathrm{T}} P_{T}=P_{T, \mathrm{FD}}^{\star}, \\
& \mathbf{1}_{m-1}^{\mathrm{T}} d=D
\end{array}
$$

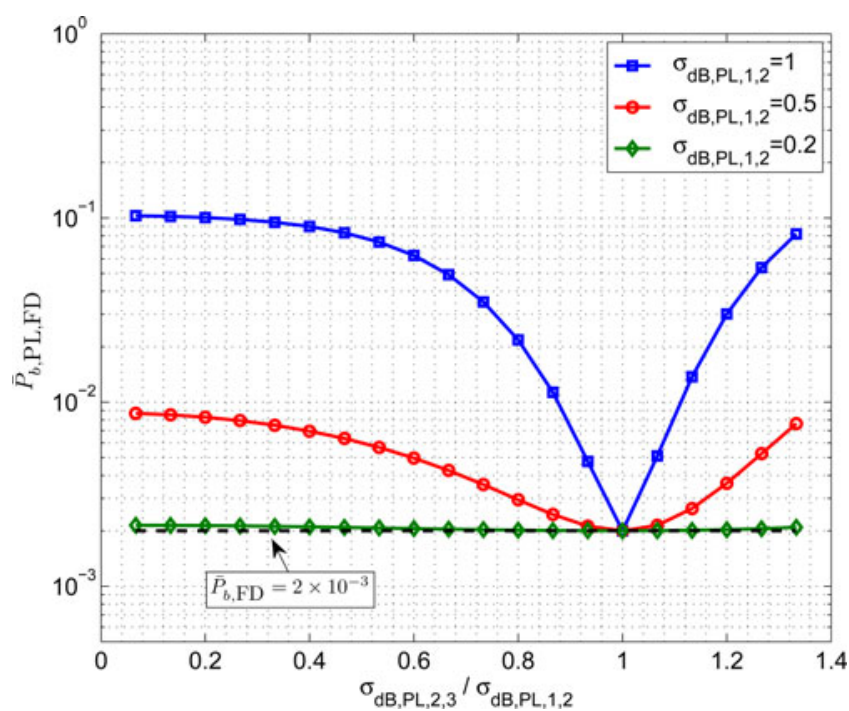

Fig. 13. Impact of taking fading into account when designing robotic routers. The figure shows that we can experience a considerable performance loss if we only consider path loss (disk models) in fading environments.

where $\gamma_{T}$ denotes the minimum acceptable SNR, which is taken to be the same for all the links, to facilitate mathematical derivations. By applying KKT conditions, optimum $\gamma_{T}$ can then be found to be

$$
\gamma_{T}^{\star}=\frac{P_{T, \mathrm{FD}}^{\star}}{D^{n}}\left(\sum_{i=2}^{m} \beta_{i-1, i}^{\frac{1}{n-1}}\right)^{n-1}
$$

Therefore, the actual performance of the path-loss-only design will be as follows, in a fading environment: $\bar{P}_{b, \mathrm{PL}, \mathrm{FD}}(\mathrm{RX})=$ $1-\prod_{i=2}^{m}\left(1-0.2\left(1+c \vartheta_{\mathrm{PL}, i-1, i} \gamma_{T}^{\star}\right)^{-\varphi_{\mathrm{PL}, i-1, i}}\right)$. Fig. 13 shows how much we lose by considering only path loss in a fading environment, for the case of one router. For this simulation, $\bar{P}_{b, 1,2, T}=\bar{P}_{b, 2,3, T}=10^{-3}, n=2$, and $c=0.5$. We have $\bar{P}_{b, \mathrm{FD}}(\mathrm{RX})=2 \times 10^{-3}$, for the design in which fading is taken into account. The figure then compares $\bar{P}_{b, \mathrm{PL}, \mathrm{FD}}(\mathrm{RX})$ with this benchmark. As can be seen, by only considering path loss, we can achieve the same performance only if $\sigma_{\mathrm{dB}, \mathrm{PL}, 1,2}=$ $\sigma_{\mathrm{dB}, \mathrm{PL}, 2,3}$. In this case, the optimum configuration of both cases will be the same. However, as we depart from this equality and as fading variances increase, we can lose orders of magnitude by not taking fading into account.

It should also be noted that the gap between the two performances will further increase if the deterministic or probabilistic path-loss/shadowing estimator of the previous section is utilized (in that case, we will also see a gap for $\sigma_{\mathrm{dB}, \mathrm{PL}, 1,2}=\sigma_{\mathrm{dB}, \mathrm{PL}, 2,3}$ ). Mathematically characterizing the performance with those estimators, however, is the subject of our future work.

\section{Robotic Router Optimization Considering both Communication and Motion Costs}

In the previous sections, we only considered the communication power consumption in the optimization of the routers. In this part, we extend our analysis and consider both communication and motion costs, in order to understand the underlying 
tradeoffs. Consider the case where the TX needs to continuously send information to the RX, during time $[0, T]$. Robotic routers at positions $x_{i}$ s, where $i \in\{2, \ldots, m-1\}$, are cooperatively relaying the information using the multihop scheme. Our goal is to minimize the overall communication and motion costs of the entire network. First, consider only the path loss. Similar to Section V-A, we assign each link a target performance requirement $\left(\gamma_{i-1, i, T}\right)$. Therefore, the required transmit power for each router is $P_{T, i-1}=\frac{\gamma_{i-1, i, T}}{\beta_{i-1, i}} d_{i-1, i}^{n}=$ $-\frac{(M-1) \ln \left(5 P_{b, i-1, i, T}\right)}{1.5 \beta_{i-1, i}} d_{i-1, i}^{n}=C_{i-1, i}\left\|x_{i}-x_{i-1}\right\|^{n}$. We then have the following optimization problem:

$$
\begin{array}{ll}
\text { minimize } & \int_{0}^{T} B \sum_{i=2}^{m-1}\left\|u_{i}\right\|^{2}+\sum_{i=2}^{m} C_{i-1, i}\left\|x_{i}-x_{i-1}\right\|^{n} d \tau \\
\text { subject to } & \dot{x}_{i}=u_{i}, \quad \forall i \in\{2, \ldots, m-1\}
\end{array}
$$

where $B$ is a positive constant (which allows the designer to properly weigh the two terms). We only consider the case where $n=2$ in this paper. The Hamiltonian of (36) will then be $\mathcal{H}=B \sum_{i=2}^{m-1}\left\|u_{i}\right\|^{2}+\sum_{i=2}^{m} C_{i-1, i}\left\|x_{i}-x_{i-1}\right\|^{2}+$ $\sum_{i=2}^{m-1} \lambda_{i}^{\mathrm{T}} u_{i}$, where $\lambda$ is the Lagrange multiplier. By applying calculus of variations [27], we can find the necessary conditions, for optimality, as follows: $\frac{\partial \mathcal{H}}{\partial u_{i}}=2 B u_{i}+\lambda_{i}=0, \frac{\partial \mathcal{H}}{\partial x_{i}}=$ $2 C_{i-1, i}\left(x_{i}-x_{i-1}\right)+2 C_{i, i+1}\left(x_{i}-x_{i+1}\right)$, and $\lambda_{i}(T)=0$ for $\forall i \in\{2, \ldots, m-1\}$. Then, the optimal control law and trajectories of the routers can be found as follows for $t \in[0, T]$ :

$$
\begin{aligned}
u^{\star}(t)= & P\left(\operatorname{diag}\left(\sqrt{\epsilon_{i}} \frac{\exp \left\{\sqrt{\epsilon_{i}} t\right\}-\exp \left\{2 \sqrt{\epsilon_{i}} T-\sqrt{\epsilon_{i}} t\right\}}{1+\exp \left\{2 \sqrt{\epsilon_{i}} T\right\}}\right)\right. \\
& \left.\otimes I_{2}\right) \times P^{-1}\left(x_{r}(0)-Q^{-1} V\right) \\
x_{r}^{\star}(t)= & P\left(\operatorname{diag}\left(\frac{\exp \left\{\sqrt{\epsilon_{i}} t\right\}+\exp \left\{2 \sqrt{\epsilon_{i}} T-\sqrt{\epsilon_{i}} t\right\}}{1+\exp \left\{2 \sqrt{\epsilon_{i}} T\right\}}\right) \otimes I_{2}\right) \\
& \times P^{-1} x_{r}(0) \\
- & P\left(\operatorname{diag}\left(\frac{\exp \left\{\sqrt{\epsilon_{i}} t\right\}+\exp \left\{2 \sqrt{\epsilon_{i}} T-\sqrt{\epsilon_{i}} t\right\}}{1+\exp \left\{2 \sqrt{\epsilon_{i}} T\right\}}-1\right)\right. \\
& \left.\otimes I_{2}\right) \times P^{-1} Q^{-1} V
\end{aligned}
$$

where

$Q=\frac{1}{B}$
$\left[\begin{array}{cccc}\sum_{i=2}^{3} C_{i-1, i} & -C_{2,3} & \ldots & 0 \\ -C_{2,3} & \sum_{i=3}^{4} C_{i-1, i} & -C_{3,4} & \vdots \\ \vdots & \vdots & \ddots & \vdots \\ 0 & \cdots & -C_{m-2, m-1} & \sum_{i=m-1}^{m} C_{i-1, i}\end{array}\right] \otimes I_{2}$

$x_{r}^{\mathrm{T}}=\left[\begin{array}{lll}x_{2}^{\mathrm{T}} & \cdots & x_{m-1}^{\mathrm{T}}\end{array}\right]^{\mathrm{T}}, \quad V=\frac{1}{B}\left[C_{1,2} x_{1}^{\mathrm{T}} \quad 0 \cdots C_{m-1, m} x_{m}^{\mathrm{T}}\right]^{\mathrm{T}}$, $P^{-1} Q P=\operatorname{diag}\left(\epsilon_{i}\right), \epsilon_{i}$ is the $i$ th eigenvalue of $Q, \otimes$ repre- sents the Kronecker product, and $\operatorname{diag}\left(\Delta_{i}\right)$ represents a diagonal matrix with $\Delta_{i} \mathrm{~s}$ on its diagonals. Since $C_{i-1, i}>0$ for $\forall i \in\{2, \ldots, m\}, Q \succ 0$.

For simplicity, consider the case where $C_{i-1, i}=C$ for all $i$. Then, $\frac{C}{B}$ denotes the ratio of communication cost to motion cost. As $\frac{C}{B} \rightarrow 0, \epsilon_{i} \rightarrow 0$, for all $i$. Then, the second term on the right-hand side of (38) goes to 0 . Hence, $x_{r}^{\star}(t) \rightarrow x_{r}(0)$, which implies that the robotic routers are more likely to stay in their initial positions and simply increase their communication power. On the other hand, as $\frac{C}{B} \rightarrow \infty, \epsilon_{i} \rightarrow \infty$, for all $i$; then the first term of (38) goes to zero, resulting in $x_{r}^{\star}(t) \rightarrow Q^{-1} V$ for $t \in(0, T]$. Next, we show that $Q^{-1} V$ is exactly the optimal solution of (29) for $n=2$, which implies that the robotic routers will move to the optimal communication configuration if the motion cost is negligible.

Lemma 3: The inverse of matrix $Q$ has the following form:

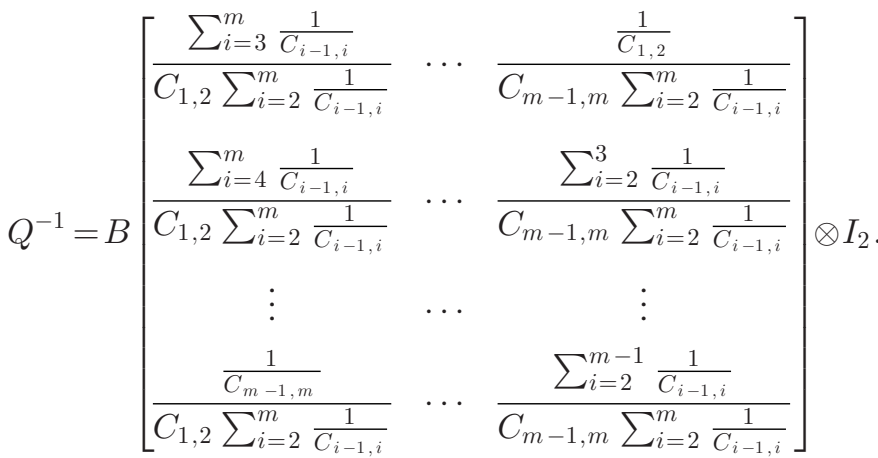

Proof: It can be easily verified that $Q^{-1} Q=I_{2 m-4}$.

By applying Lemma 3, we then have

$$
x_{i}^{\star}(t) \rightarrow \frac{\sum_{j=i+1}^{m} \frac{1}{C_{j-1, j}}}{\sum_{j=2}^{m} \frac{1}{C_{j-1, j}}} x_{1}+\frac{\sum_{j=2}^{i} \frac{1}{C_{j-1, j}}}{\sum_{j=2}^{m} \frac{1}{C_{j-1, j}}} x_{m}
$$

if $\frac{C}{B} \rightarrow \infty$, i.e., if the motion cost is negligible. This means that the routers should be on the line segment from the transmitter to the receiver, with the following optimum distances:

$$
\left\|x_{i}-x_{i-1}\right\|=\frac{\frac{1}{C_{i-1, i}}}{\sum_{j=2}^{m} \frac{1}{C_{j-1, j}}}\left\|x_{m}-x_{1}\right\|=\frac{\frac{\beta_{i-1, i}}{\gamma_{i-1, i, T}}}{\sum_{j=2}^{m} \frac{\beta_{j-1, j}}{\gamma_{j-1, j, T}}} D .
$$

As can be seen, this is exactly what we found in Proposition 4 for $n=2$.

Fig. 14 shows the communication and motion tradeoffs for two cases. In the first case, we have $M=4, P_{b, i-1, i, T}=10^{-3}$, $\beta_{i-1, i}=10^{-3}$ for all $i, T=10$, and $B=5 \times 10^{6}$, resulting in $\frac{C}{B}=2.1 \times 10^{-3}$, which means that the communication cost is low, as compared with motion cost. In the second case, we have $M=16, P_{b, i-1, i, T}=10^{-6}, \beta_{i-1, i}=10^{-3}$, for all $i, T=10$ and $B=10^{3}$, resulting in $\frac{C}{B}=122.1$, i.e., the communication cost is high as compared with the motion cost. As can be seen, when the communication cost is low, all the routers will stay close to the initial positions. On the hand, when the communication cost is high, all the routers will move to the solution of (29).

1) Extension to Fading Environments: We can readily extend the framework of (36) to fading environments by considering the probabilistic estimator of (21). Similar to Section V-B, 


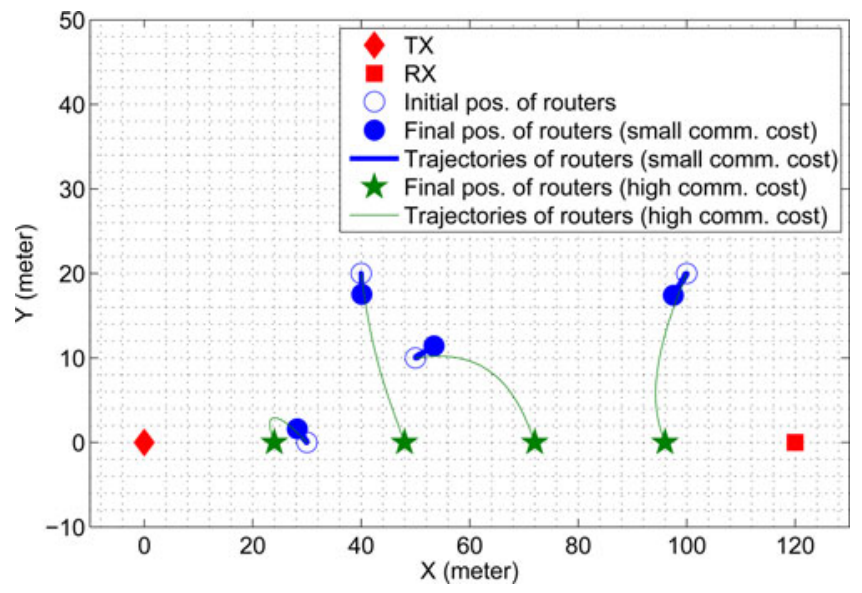

Fig. 14. Communication and motion tradeoffs in robotic routers.

we assign each link a minimum target average performance requirement $\left(\Upsilon_{\mathrm{PL}, i-1, i, T}\right)$. Then, the required transmit power for each router is $P_{T, i-1}=\frac{\Upsilon_{\mathrm{PL}, i-1, i, T}}{\beta_{i-1, i}} d_{i-1, i}^{n}=\widetilde{C}_{i-1, i} d_{i-1, i}^{n}$. We then have the following optimization framework:

$$
\begin{array}{ll}
\operatorname{minimize} & \int_{0}^{T} B \sum_{i=2}^{m-1}\left\|u_{i}\right\|^{2}+\sum_{i=2}^{m} \widetilde{C}_{i-1, i}\left\|x_{i-1}-x_{i}\right\|^{n} d \tau \\
\text { subject to } & \dot{x}_{i}=u_{i} \quad \forall i \in\{2, \ldots, m-1\} .
\end{array}
$$

As can be seen, all the analysis in this section holds for the case of $n=2$. Because of fading, however, $\widetilde{C}_{i-1, i}$ is larger than $C_{i-1, i}$ if we take $\bar{P}_{b, i-1, i, T}=P_{b, i-1, i, T}$. This implies that communication is more costly in fading environments, as expected. In addition, as $\sigma_{\mathrm{dB}, \mathrm{PL}, i-1, i} \rightarrow 0$, we have $\widetilde{C}_{i-1, i} \rightarrow C_{i-1, i}$, i.e., the solution of fading case converges to the path-loss case if the variance of fading goes to 0 . Note that only the probabilistic path-loss estimator approach is considered in (40). Hence, the performance can further be improved if the deterministic or probabilistic path-loss/shadowing estimator is utilized instead. Solving (40) for such cases, however, is among our future work.

\section{FURTHER EXTENSIONS-PRELIMINARY EXPERIMENTAL RESULTS AND THE IMPACT OF LOCALIZATION ERRORS}

In Figs. 10 and 11, we simulated a robotic operation, using the real channel data of Fig. 9. In this section, we show a preliminary robotic operation, where a robot tries to maintain the connectivity of two stationary homogeneous routers in our basement. We emphasize that this is a preliminary test, with the main goal to explore the impact of localization errors. In particular, we discuss interesting interplays between the localization quality and the channel correlation/learning quality. Fig. 15 shows the experimental setup, where the positions of the TX and RX routers are marked. The right side of Fig. 9 shows a color map of the received communication signal strength, from the TX, in this environment. We use the Pioneer robot of the left side of Fig. 9 as a robotic router. The initial position of the robot is marked with an empty circle on the right side of Fig. 15. The robot uses a priori channel measurements that are collected in this environment, for motion planning, mounting to $1.04 \%$ to
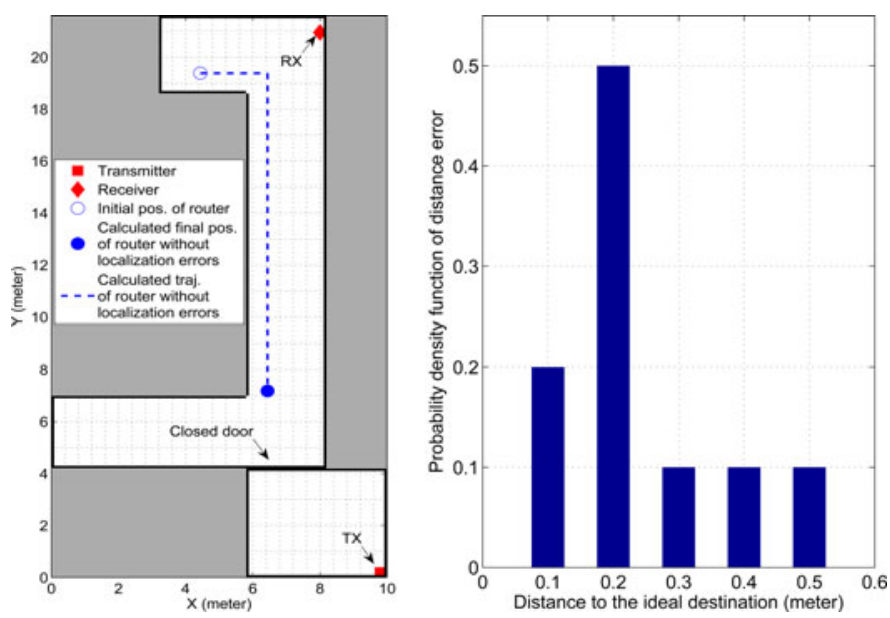

Fig. 15. (Left) Initial position, calculated trajectory, and calculated final position of the router, by using our proposed optimization framework and probabilistic path-loss/shadowing estimator. (Right) Pdf of the distance of the true final position of the robot to the calculated final position, after running the experiment ten times, from the same initial position. The error is mainly due to localization errors.

the TX and $1.24 \%$ to the RX. By using the probabilistic channel estimator of Section IV-A (see Lemmas 1 and 2), the robot then predicts the channel at unvisited locations. ${ }^{9}$ It then finds its optimum position by solving the probabilistic optimization problem of (27), with the probabilistic path-loss/shadowing estimator, through an exhaustive search.

The full circle on the left side of Fig. 15 shows the optimum position. As for motion planning, a simple strategy is used that finds a route that is parallel to the walls, with minimum turns. The left side of Fig. 15 shows the calculated route. The true route and final position, however, will be different, due to localization errors. The robot uses onboard gyroscope and wheel encoders for localization. Since this area of our basement is sloped, we also have a simple compensating function to control the direction. More sophisticated localization sensors/algorithms can also be used to improve the performance [28]-[30]. The right side of Fig. 15 shows the pdf of the distance of the true final position of the robot to the calculated optimum final position [see the filled circle on the left side of Fig. 15], after running the experiment for ten times.

The top of Fig. 16 shows the signal strength from the TX and $\mathrm{RX}$, along the trajectory, while the bottom of Fig. 16 shows the simulated end-to-end BER, which is based on the measurements of signal strength. The curves are averaged over ten instants of running the experiment from the same initial position. As can be seen, the signal strength from the TX is low at the beginning, which results in a high BER. As the robot moves toward the final position, the signal strength from the TX increases and the end-to-end BER decreases a few orders of magnitude. The

\footnotetext{
${ }^{9}$ In general, localization errors can directly affect the channel assessment quality. Typically, however, channel assessment is mainly based on a very few measurements that are gathered at the beginning of the operation. Localization errors have not yet accumulated that much at the beginning of the operation, assuming that the initial positions of the robots are known. Thus, its impact on channel learning may be negligible, as compared with the impact of channel parameter estimation errors or other channel learning uncertainties.
} 

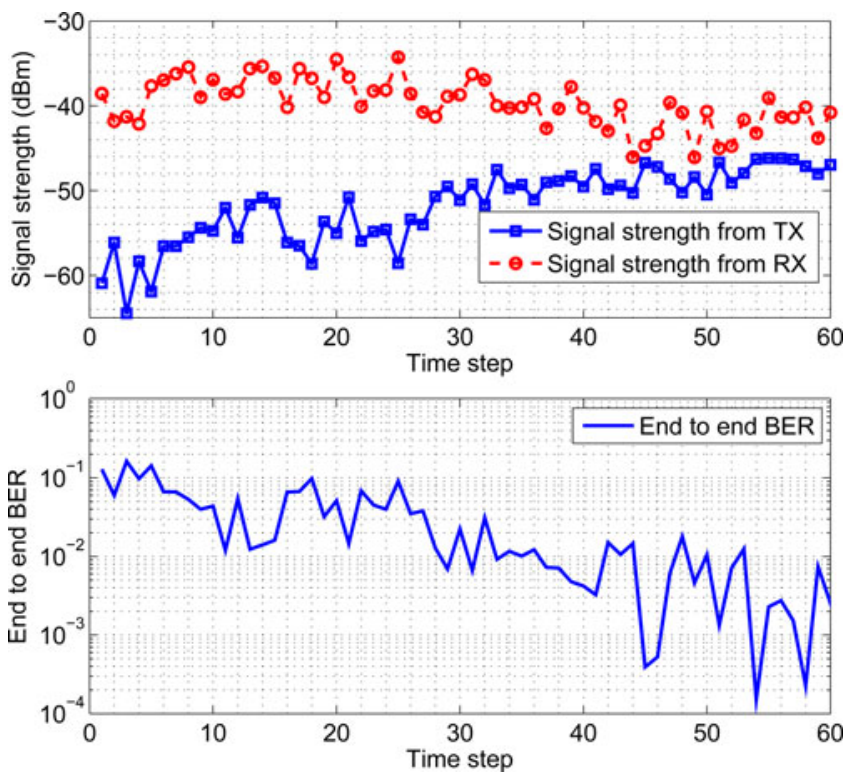

Fig. 16. Signal strength from TX/RX along the trajectory (top) and the simulated end-to-end BER, which is based on the signal strength measurements (bottom).

fluctuations in the curves are caused by multipath fading. It is worth noting that the optimum position of the router, which is based on the sparse samples of the channels, is close to the TX. This makes sense as the closed door makes communication with the TX harder.

\section{A. Interplay Between Localization Quality and Channel Correlation/Learning Quality}

Localization errors can impact the optimum positioning of the routers, affecting the overall performance. However, the level of impact depends on the channel parameters. First, consider the case where the channel is dominated by multipath fading. Since our channel estimator (or any estimator for that matter) does not predict the multipath fading component, the final calculated optimum configuration does not experience the planned channel qualities. The true performance at that configuration would be the same as any other configuration in its vicinity, due to multipath fading. Thus, the impact of multipath fading is similar to having localization errors in this case. In other words, localization errors do not impact the performance significantly. On the other hand, consider the case where multipath fading is negligible such that channel can be assessed almost perfectly. First, consider the case where shadowing decorrelation distance is large (channel stays correlated over a large distance). Then, small localization errors do not impact the performance that much since the true final position experiences highly correlated channels with the calculated final position. However, if the decorrelation distance is small, localization errors can impact the performance. In summary, if the localization errors are small, with respect to the decorrelation distance, their impact on the performance of our proposed framework becomes negligible. Furthermore, as multipath fading increases, for any given localization quality and decorrelation distance, the impact of localization errors becomes smaller.

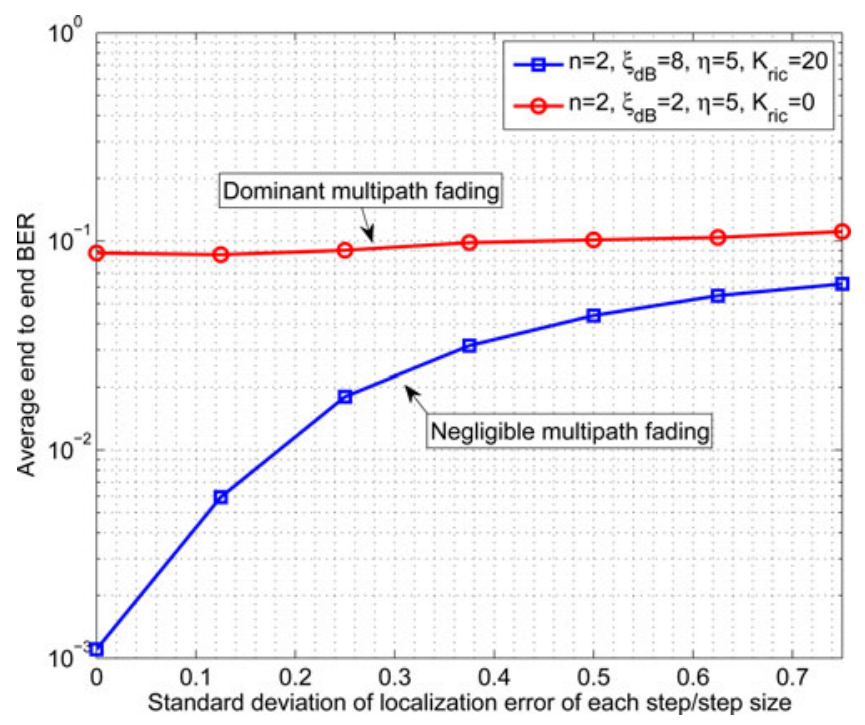

Fig. 17. Interplay between localization quality and channel correlation/ learning quality.

The simulation results of Fig. 17 verify our hypothesis, for the case where one router tries to optimize the connectivity of two fixed nodes. In this simulation, the workspace is taken to be $40 \mathrm{~m} \times 40 \mathrm{~m}$, and the step size of the robot is $0.4 \mathrm{~m}$. The localization error is modeled as an additive zero-mean Gaussian noise [30]. We assume that the robot has 5\% a priori channel samples to predict the channel, using our probabilistic path-loss/shadowing estimator. The simulation is averaged over several runs of different channel samples (same underlying parameters) and start positions. The figure shows two curves. For the case of $\xi_{\mathrm{dB}}=8$ and $K_{\text {ric }}=20$, multipath fading is negligible. The curve then shows that localization errors impact the performance more drastically as the localization error increases. For the case of $\xi_{\mathrm{dB}}=2$ and $K_{\text {ric }}=0$, on the other hand, the channel is dominated by multipath fading. Then, the performance stays very similar to the case of no localization error, i.e., the performance is not as sensitive to localization errors. Further characterization of the interplay between the two, as well as understanding the direct impact of localization errors on channel learning, are subjects of our future work.

\section{CONCLUSION}

In this paper, we have considered the problem of robotic router formation, where two nodes need to maintain their connectivity by using a number of robotic routers. Instead of optimizing the formation of the routers by maximizing the Fiedler eigenvalue, we took a different approach and considered the true reception quality (BER) as a performance metric. We showed how utilizing this metric results in a different robotic configuration, with a considerably better performance. We furthermore extended our results to fading environments. We proposed a probabilistic router formation framework by integrating our previously proposed probabilistic channel prediction approach with robotic router optimization. We showed that our framework can improve the performance considerably, as compared 
with only considering disk models for communication. We also considered power limitations of the network, including both communication and motion costs, and characterized the underlying tradeoffs. Finally, we showed the performance with a simple preliminary robotic experiment, with an emphasis on the impact of localization errors. Along this line, we discussed interesting interplays between the localization quality and the channel correlation/learning quality.

\section{APPENDIX}

Here, we extend Propositions 1 and 3 to fading environments. Specifically, we characterize the properties of the optimization problem of (27), for the case of probabilistic path-loss estimator, i.e., $\Upsilon_{\mathrm{dB}, i-1, i}=\Upsilon_{\mathrm{dB}, \mathrm{PL}, i-1, i}$ and $\sigma_{\mathrm{dB}, i-1, i}=\sigma_{\mathrm{dB}, \mathrm{PL}, i-1, i}$. We then have the following optimization problem:

maximize $\overline{\mathcal{J}}\left(x_{r}\right)$

$$
=\sum_{i=2}^{m} \ln \left(1-0.2\left(1+c \vartheta_{\mathrm{PL}, i-1, i} \Upsilon_{\mathrm{PL}, i-1, i}\right)^{-\varphi_{\mathrm{PL}, i-1, i}}\right)
$$

subject to $\quad x_{i} \in \mathcal{W}, \quad \forall i \in\{2, \ldots, m-1\}$

where $\varphi_{\mathrm{PL}, i-1, i}=\left(\exp \left\{\left(a \sigma_{\mathrm{dB}, \mathrm{PL}, i-1, i}\right)^{2}\right\}-1\right)^{-1}, \vartheta_{\mathrm{PL}, i-1, i}$ $=\exp \left\{1.5\left(a \sigma_{\mathrm{dB}, \mathrm{PL}, i-1, i}\right)^{2}\right\}-\exp \left\{0.5\left(a \sigma_{\mathrm{dB}, \mathrm{PL}, i-1, i}\right)^{2}\right\}$, and $a=\frac{\ln 10}{10}$.

Proposition 5: If $\sigma_{\mathrm{dB}, \mathrm{PL}, i-1, i}<\frac{1}{a} \sqrt{\ln (n+1)}$ and $\Upsilon_{\mathrm{PL}, i-1, i}$ $\geq \frac{n+1}{n c}\left(\varphi_{\mathrm{PL}, i-1, i} \vartheta_{\mathrm{PL}, i-1, i}-\frac{\vartheta_{\mathrm{PL}, i-1, i}}{n}\right)^{-1}$ all the time, then the optimization problem of (41) is concave for a convex $\mathcal{W}$.

Proof: Let $\overline{\mathcal{J}}_{i}\left(x_{r}\right)=\ln \left(1-0.2\left(1+c \vartheta_{\mathrm{PL}, i-1, i}\right.\right.$ $\left.\left.\Upsilon_{\mathrm{PL}, i-1, i}\right)^{-\varphi_{\mathrm{PL}, i-1, i}}\right)$. Similar to Proposition 1, it is sufficient to force $\frac{\partial^{2} \overline{\mathcal{J}}_{i}}{\partial \Upsilon_{\mathrm{PL}, i-1, i}^{2}}+\frac{\partial \overline{\mathcal{J}}_{i}}{\partial \Upsilon_{\mathrm{PL}, i-1, i}} \frac{n+1}{n \Upsilon_{\mathrm{PL}, i-1, i}} \leq 0$, for all $i$, to guarantee the concavity, which results in the following sufficient condition: $\Upsilon_{\mathrm{PL}, i-1, i} \geq \frac{n+1}{n c}\left(\varphi_{\mathrm{PL}, i-1, i} \vartheta_{\mathrm{PL}, i-1, i}-\frac{\vartheta_{\mathrm{PL}, i-1, i}}{n}\right)^{-1}$, if $\sigma_{\mathrm{dB}, \mathrm{PL}, i-1, i}<\frac{1}{a} \sqrt{\ln (n+1)}$.

If there is no obstacle on the line segment between the transmitter and the receiver, Proposition 2 clearly holds, resulting in the following simplified optimization problem, on the line segment between the transmitter and the receiver:

$$
\begin{array}{ll}
\operatorname{maximize} & \overline{\mathcal{J}}(d)= \\
& \sum_{i=2}^{m} \ln \left(1-0.2\left(1+c \vartheta_{\mathrm{PL}, i-1, i} \Upsilon_{\mathrm{PL}, i-1, i}\right)^{-\varphi_{\mathrm{PL}, i-1, i}}\right)
\end{array}
$$

subject to $\quad d_{i-1, i} \geq 0, \forall i \in\{2, \ldots, m\}, \mathbf{1}_{m-1}^{T} d=D$.

Proposition 6: Assume that the concavity condition in Proposition 5 holds. Then, the optimal solution of (42) satisfies the following properties.

1) If $\alpha_{i-1, i}>\alpha_{j-1, j}$ and $\sigma_{\mathrm{dB}, \mathrm{PL}, i-1, i}=\sigma_{\mathrm{dB}, \mathrm{PL}, j-1, j}$, then $d_{i-1, i}^{\star}>d_{j-1, j}^{\star}$

2) If $\quad \alpha_{i-1, i}=\alpha_{j-1, j}$, $\underline{\exp \left\{1.5 \exp \left\{\left(a \sigma_{\mathrm{dB}, \mathrm{PL}, i-1, i}\right)^{2}\right\}-0.5\right\}-1}$ $\frac{\exp \left\{1.5 \exp \left\{\left(a \sigma_{\mathrm{dB}, \mathrm{PL}, j-1, j}\right)^{2}\right\}-0.5\right\}-1}{c \vartheta_{\mathrm{PL}, j-1, j}}$, and $\sigma_{\mathrm{dB}, \mathrm{PL}, i-1, i}>$ $\sigma_{\mathrm{dB}, \mathrm{PL}, j-1, j}$, then $d_{i-1, i}^{\star}<d_{j-1, j}^{\star} ;$
3) If $\alpha_{i-1, i}=\alpha_{j-1, j}$ and $\sigma_{\mathrm{dB}, \mathrm{PL}, i-1, i}=\sigma_{\mathrm{dB}, \mathrm{PL}, j-1, j}$, then $d_{i-1, i}^{\star}=d_{j-1, j}^{\star}$

where $d_{i-1, i}^{\star}$ is the optimum distance between nodes $i-1$ and $i$.

Proof: Similar to Proposition 3, the optimal solution of (42) satisfies the KKT conditions, which results in the following:

$$
\frac{\partial \overline{\mathcal{J}}_{i}\left(d^{\star}\right)}{\partial d_{i-1, i}^{\star}}=\frac{\partial \overline{\mathcal{J}}_{j}\left(d^{\star}\right)}{\partial d_{j-1, j}^{\star}}
$$

Parts 1 and 3 can then be proved, similar to Proposition 3. For the proof of Part 2, let $\frac{\partial \bar{J}_{i}}{\partial \Upsilon_{\mathrm{PL}, i-1, i}}=\frac{0.2 c}{f_{i-1, i}}$, where $f_{i-1, i}=\exp$ $\left\{-0.5\left(a \sigma_{\mathrm{dB}, \mathrm{PL}, i-1, i}\right)^{2}\right\}\left(\left(1+c \vartheta_{\mathrm{PL}, i-1, i} \Upsilon_{\mathrm{PL}, i-1, i}\right)^{\varphi_{\mathrm{PL}, i-1, i}+1}\right.$ $\left.-0.2\left(1+c \vartheta_{\mathrm{PL}, i-1, i} \Upsilon_{\mathrm{PL}, i-1, i}\right)\right)$. Then, $\frac{\partial f_{i-1, i}}{\partial \sigma_{\mathrm{dB}, \mathrm{PL}, i-1, i}}<$ $a^{2}\left(1+c \vartheta_{\mathrm{PL}, i-1, i} \Upsilon_{\mathrm{PL}, i-1, i}\right)^{\varphi_{\mathrm{PL}, i-1, i}+1} \varphi_{\mathrm{PL}, i-1, i}^{2} \exp \{0.5$ $\left.\left(a \sigma_{\mathrm{dB}, \mathrm{PL}, i-1, i}\right)^{2}\right\} \sigma_{\mathrm{dB}, \mathrm{PL}, i-1, i}\left(3 \exp \left\{\left(a \sigma_{\mathrm{dB}, \mathrm{PL}, i-1, i}\right)^{2}\right\}-1-\right.$ $\left.2 \ln \left(1+c \vartheta_{\mathrm{PL}, i-1, i} \Upsilon_{\mathrm{PL}, i-1, i}\right)\right)$. Therefore, $\frac{\partial f_{i-1, i}}{\partial \sigma_{\mathrm{dB}, \mathrm{PL}, i-1, i}}$ is negative if

$$
\Upsilon_{\mathrm{PL}, i-1, i} \geq \frac{\exp \left\{1.5 \exp \left\{\left(a \sigma_{\mathrm{dB}, \mathrm{PL}, i-1, i}\right)^{2}\right\}-0.5\right\}-1}{c \vartheta_{\mathrm{PL}, i-1, i}} .
$$

If (44) is satisfied for $i$ and $j$, then $\frac{\partial \overline{\mathcal{J}}_{i}}{\partial \Upsilon_{\mathrm{PL}, i-1, i}}$ and $\frac{\partial \overline{\mathcal{J}}_{j}}{\partial \Upsilon_{\mathrm{PL}, j-1, j}}$ are monotonically increasing, with respect to $\sigma_{\mathrm{dB}, \mathrm{PL}, i-1, i}$ and $\sigma_{\mathrm{dB}, \mathrm{PL}, j-1, j}$, respectively. Assume that the optimal solution of (42) is $d_{i-1, i}^{\star}=d_{j-1, j}^{\star}$ if $\alpha_{i-1, i}=\alpha_{j-1, j}$ and $\sigma_{\mathrm{dB}, \mathrm{PL}, i-1, i}>$ $\sigma_{\mathrm{dB}, \mathrm{PL}, j-1, j}$. Then, $\frac{\partial \overline{\mathcal{J}}_{i}\left(d^{\star}\right)}{\partial d_{i-1, i}^{\star}}<\frac{\partial \overline{\mathcal{J}}_{j}\left(d^{\star}\right)}{\partial d_{j-1, j}^{\star}}$. Therefore, (43) can only be achieved by increasing $\frac{\partial \overline{\mathcal{J}}_{i}\left(d^{\star}\right)}{\partial d_{i-1, i}^{\star}}$ or equivalently decreasing $\frac{\partial \overline{\mathcal{J}}_{j}\left(d^{\star}\right)}{\partial d_{j-1, j}^{\star}}$. By the concavity condition, we know that $\frac{\partial \overline{\mathcal{J}}_{i}(d)}{\partial d_{i-1, i}}$ is decreasing with respect to $d_{i-1, i}$. Hence, the optimal configuration is achieved iff $d_{i-1, i}^{\star}<d_{j-1, j}^{\star}$.

Remark 7: As compared with the conditions of $\gamma_{i-1, i}$ in Propositions 1 and 3, the sufficient conditions of Propositions 5 and 6 not only require that the average SNR (distance-dependent path loss) is above the minimum level but need the variations of fading around this average to be small as well. As an example, consider the real channel measurements of Fig. 9 in our basement, where the estimated path-loss exponent is $\hat{n}=$ 2.32, and the standard deviation of fading is $\sigma_{\mathrm{dB}, \mathrm{PL}, i-1, i}=$ 4.66. The first condition of Proposition 5 is then satisfied since $\sigma_{\mathrm{dB}, \mathrm{PL}, i-1, i}<\frac{1}{a} \sqrt{\ln (2.32+1)}$. The second condition of Proposition 5, as well as the second condition of Part 2 of Proposition 6 , can further be satisfied if $\Upsilon_{\mathrm{dB}, \mathrm{PL}, i-1, i} \geq 13.58 \mathrm{~dB}$, which, for instance, happens in a continuous area around the transmitter that mounts to $44.6 \%$ of the whole basement. Thus, with the proper use of a couple of routers, the required conditions can be satisfied, depending on the initial positions of the routers.

The first and third parts of Proposition 6 show similar properties to Proposition 3. In fading environments, average BER is also monotonically increasing with $\sigma_{\mathrm{dB}, \mathrm{PL}, i-1, i}$, i.e., the fading level of the channel. The second part of Proposition 6 then shows that in order to compensate for the performance degradation in the links with larger $\sigma_{\mathrm{dB}, \mathrm{PL}, i-1, i} \mathrm{~s}$, the routers should form a configuration in which the distances of those links are smaller. 


\section{REFERENCES}

[1] Y. Yan and Y. Mostofi, "Robotic router formation-A bit error rate approach," in Proc. IEEE Military Commun. Conf., San Jose, CA, Nov. 2010, pp. 1287-1292.

[2] Y. Kim and M. Mesbahi, "On maximizing the second smallest eigenvalue of a state-dependent graph Laplacian," IEEE Trans. Automat. Control, vol. 51, no. 1, pp. 116-120, Apr. 2006.

[3] M. C. De Gennaro and A. Jadbabaie, "Decentralized control of connectivity for multi-agent systems," in Proc. 45th IEEE Conf. Decision Control, San Diego, CA, Dec. 2006, pp. 3628-3633.

[4] M. M. Zavlanos and G. J. Pappas, "Potential fields for maintaining connectivity of mobile networks," IEEE Trans. Robot., vol. 23, no. 4, pp. 812816, Aug. 2007

[5] M. M. Zavlanos and G. J. Pappas, "Controlling connectivity of dynamic graphs," in Proc. 44th IEEE Conf. Decision Control and Eur. Control Conf., Seville, Spain, Dec. 2005, pp. 6388-6393.

[6] E. Stump, A. Jadbabaie, and V. Kumar, "Connectivity management in mobile robot teams," in Proc. 48th IEEE Int. Conf. Robot. Autom., Pasadena, CA, May 2008, pp. 1525-1530.

[7] O. Tekdas, W. Yang, and V. Isler, "Robotic routers: Algorithms and implementation," Int. J. Robot. Res., vol. 29, no. 1, pp. 110-126, Jan. 2010.

[8] C. Dixon and E. W. Frew, "Maintaining optimal communication chains in robotic sensor networks using mobility control," presented at the 1st Int. Conf. Robot Commun. Coordination, Athens, Greece, Oct. 2007.

[9] D. Tardioli, A. R. Mosteo, L. Riazuelo, J. L. Villarroel, and L. Montano, "Enforcing network connectivity in robot team missions," Int. J. Robot. Res., vol. 29, no. 4, pp. 460-480, Apr. 2010.

[10] D. K. Goldenberg, J. Lin, S. A. Morse, B. E. Rosen, and R. Y. Yang, "Towards mobility as a network control primitive," in Proc. 5th ACM Int. Symp. MobiHoc, 2004, pp. 163-174.

[11] S. Yu and C. S. G. Lee, "Lifetime maximization in mobile sensor networks with energy harvesting," in Proc. 51 th IEEE Int. Conf.Robot. Autom., May 2011, pp. 131-142.

[12] K. Xu, H. Hassanein, G. Takahara, and Q. Wang, "Relay node deployment strategies in heterogeneous wireless sensor networks," IEEE Trans. Mobile Comput., vol. 9, no. 2, pp. 145-159, Feb. 2010

[13] W. Wang and V. Srinivasan, K.-C. Chua, "Extending the lifetime of wireless sensor networks through mobile relays," IEEE Trans. Network., vol. 16, no. 5, pp. 1108-1120, Oct. 2008.

[14] J. Laneman, D. Tse, and G. Wornell, "Cooperative diversity in wireless networks: Efficient protocols and outage behavior," IEEE Trans. Inf. Theory, vol. 50, no. 12, pp. 3062-3080, Dec. 2004

[15] A. Bletsas, A. Khisti, D. P. Reed, and A. Lippman, "A simple cooperative diversity method based on network path selection," IEEE J. Sel. Areas Commun., vol. 24, no. 3, pp. 659-672, Mar. 2006.

[16] Y. Mostofi, M. Malmirchegini, and A. Ghaffarkhah, "Estimation of communication signal strength in robotic networks," in Proc. 50th IEEE Int. Conf. Robot. and Autom., Anchorage, AK, May 2010, pp. 1946-1951.

[17] M. Malmirchegini and Y. Mostofi, "On the spatial predictability of communication channels," IEEE Trans. Wireless Commun., vol. 11, no. 3, pp. 964-978, Mar. 2012.

[18] A. Goldsmith, Wireless Communications. Cambridge, U.K.: Cambridge Univ. Press, 2005.

[19] J. G. Proakis, Digital Communications, 4th ed. New York: McGraw-Hill, 2001.

[20] D. B. Johnson and D. A. Maltz, Dynamic Source Routing in Ad Hoc Wireless Networks, vol. $353 . \quad$ Norwell, MA: Kluwer, 1996, ch. 5, pp. 153181

[21] C. E. Perkins and E. M. Royer, "Ad-hoc on-demand distance vector routing," in Proc. 2nd IEEE Workshop Mobile Comput. Syst. Appl., New Orleans, LA, Feb. 1999, pp. 90-100.

[22] S. Boyd and L. Vandenberghe, Convex Optimization. Cambridge, U.K.: Cambridge Univ. Press, 2004.

[23] Y. Mostofi, A. Gonzalez-Ruiz, A. Ghaffarkhah, and D. Li, "Characterization and modeling of wireless channels for networked robotic and control systems-A comprehensive overview," in Proc. IEEE/RSJ Int. Conf. Robots Syst., St. Louis, MO, Oct. 2009, vol. 3, pp. 4849-4854.
[24] C. Oestges, N. Czink, B. Bandemer, P. Castiglione, F. Kaltenberger, and A. J. Paulraj, "Experimental characterization and modeling of outdoorto-indoor and indoor-to-indoor distributed channels," IEEE Trans. Veh. Technol., vol. 59, no. 5, pp. 2253-2265, Jun. 2010.

[25] H. Hashemi, "A study of temporal and spatial variations of the indoor radio propagation channel," in Proc. 5th IEEE Int. Symp. Pers., Indoor Mobile Radio Comm., Sep. 1994, vol. 1, pp. 127-134.

[26] A. Abdi and M. Kaveh, "On the utility of gamma pdf in modeling shadow fading (slow fading)," in Proc. 49th IEEE Veh. Technol. Conf., Houston, TX, Jul.1999, vol. 3, pp. 2308-2312.

[27] R. E. Kirk, Optimal Control Theory: An Introduction. New York: Dover, 2004.

[28] D. Fox, W. Burgard, and S. Thrun, "Markov localization for mobile robots in dynamic environments," J. Artif. Intell. Res., vol. 11, pp. 391-427, 1999.

[29] S. Thrun, W. Burgard, and D. Fox, Probabilistic Robotics. Cambridge, MA: MIT Press, 2005.

[30] J. J. Leonard and H. F. Durrant-Whyte, Directed Sonar Sensing for Mobile Robot Navigation. $\quad$ New York: Springer-Verlag, 1992.

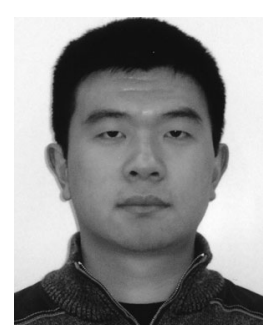

Yuan Yan received the B.S. and M.S. degrees from Huazhong University of Science and Technology, Wuhan, China, in 2006 and 2008, respectively. He is currently working towards the Ph.D. degree with the Department of Electrical and Computer Engineering, University of California, Santa Barbara.

His research interests include robotic router networks and communication-aware motion planning.

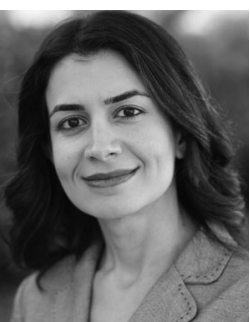

Yasamin Mostofi (M'04) received the B.S. degree in electrical engineering from the Sharif University of Technology, Tehran, Iran, in 1997 and the M.S. and $\mathrm{Ph} . \mathrm{D}$. degrees in the area of wireless communication systems from Stanford University, Stanford, CA, in 1999 and 2004, respectively.

She is currently an associate professor with the Department of Electrical and Computer Engineering, University of California, Santa Barbara. Prior to that, she was an assistant professor with the University of New Mexico. She was a postdoctoral scholar in control and dynamical systems with the California Institute of Technology from 2004 to 2006

Dr. Mostofi received the Presidential Early Career Award for Scientists and Engineers (PECASE) and the U.S. National Science Foundation (NSF) CAREER award. She also received the Bellcore fellow-advisor award from the Stanford Center for Telecommunications in 1999. She won the 2008-2009 Electrical and Computer Engineering Distinguished Researcher Award from the University of New Mexico. Her current research lies at the intersection of the two areas of communications and control/robotics in mobile sensor networks. Current research projects include communication-aware navigation and decision making in robotic networks, compressive sensing and control, obstacle mapping, robotic routers, and cooperative information processing. She has served on the Control Systems Society conference editorial board since 2008 